\title{
An elasto-plastic damage model for argillaceous geomaterials
}

\author{
A. Alizadeh ${ }^{1}$ and B. Gatmiri ${ }^{1,2}$
}

\begin{abstract}
The aim of this paper is to present a new elastoplastic damage model for unsaturated porous media. The proposed model within the context of poromechanics is intended for constitutive modelling of geomaterials which show two (irreversible) dissipative aspects involving plastic flow and damage. Independent stress state variables, namely net stress and suction, are adopted as stress variables of the model. Barcelona Basic Model is extended to a nonisothermal formulation and is coupled to a damage formulation using damage dependent yield surface and damaged stress variables. The stress state variables are transformed into the damaged state through the definition of effective damaged suction and effective damaged net stress in the framework of Continuum Damage Mechanics (CDM). Damage effects on intrinsic permeability and transfer laws are also taken into consideration. The model has been implemented in $\Theta$-STOCK Code and predictive capabilities of the model have been evaluated against experimental results from the literature. The model is validated by comparing the numerical results with experimental results of argillites and results of an elastodamage model. The proposed model is also shown to give satisfactory simulation results which match the experimental data from a heating test on bentonite samples.
\end{abstract}

Keywords: Clayey geomaterials; continuum damage mechanics (CDM);

unsaturated porous media; heating of bentonite

\section{Nomenclature}

$C_{0} \quad$ material parameter

$C_{1} \quad$ material parameter

\footnotetext{
${ }^{1}$ Civil Engineering Department, University of Tehran, Tehran, Iran

${ }^{2}$ Agence Nationale pour la gestion des Dchets RAdioactifs (ANDRA), France
} 
$C_{a} \quad$ material parameter

$C_{P W} \quad$ water specific heat capacity

$C_{P V} \quad$ vapour specific heat capacity

$C_{P a} \quad$ gas specific heat capacity

$e \quad$ void ratio

$F_{p} \quad$ plastic yield surface

$F_{d} \quad$ damage yield surface

g material parameter

$H \quad$ Henry's coefficient

$h_{f g} \quad$ latent heat of vaporization

Id identity matrix

$\mathbf{K}_{\text {int }}^{\text {tot }}$ intrinsic permeability tensor

$K_{r t} \quad$ relative thermal permeability

$K_{r c} \quad$ relative capillary permeability

$M \quad$ slope of critical state line

$n$ porosity

$p \quad$ mean net stress

$\hat{p} \quad$ damaged effective mean net stress

$p_{\text {geo }} \quad$ geostatic stress

$p_{\text {ref }} \quad$ reference stress

$p_{s} \quad$ intercept of plastic yield surface

$\hat{p}_{s} \quad$ damaged counterpart of $p_{s}$

$Q \quad$ total heat flow

$q \quad$ deviatoric stress

$\hat{q} \quad$ damaged deviatoric stress

$s \quad$ matric suction

$\hat{S} \quad$ damaged effective suction

$G \quad$ Gibbs free energy

r material parameter

$S_{r}, S_{w} \quad$ liquid degree of saturation

$T$ temperature

$t, t^{\prime} \quad$ time

$u_{a} \quad$ air pressure

$u_{w} \quad$ water pressure

$\alpha_{0}^{*}, \alpha_{1}$ material parameter

$\alpha_{2}, \alpha_{3}$ material parameter

$\alpha_{a} \quad$ material parameter

$\alpha_{w} \quad$ material parameter 


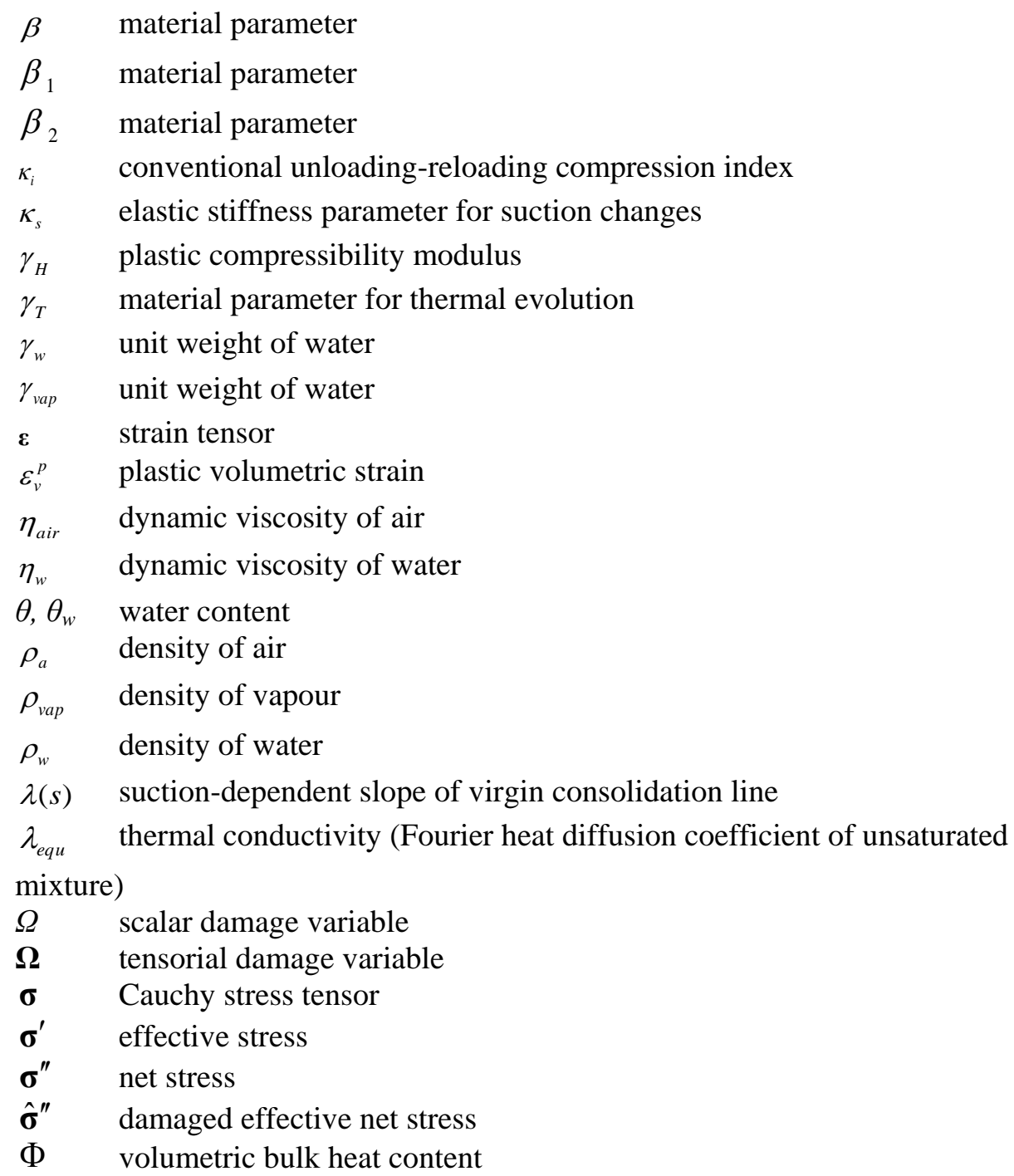




\section{Introduction}

Nonlinearity of behaviour of geomaterials was traditionally attributed to slippage and dislocation of particles of porous media skeleton and was therefore modelled using mere elastoplastic models. Based on this hypothesis, many models have been developed for geomaterials (Alonso et al., 1990; Gallipoli et al., 2003; Wheeler et al., 2003; Shuku et al. 2012; Xiong et al. 2014 among others) and they have widely been employed either for research or for practical engineering purposes. After a long period of neglect, recently there has been increasing interest in the modelling of damage in geotechnical engineering and some damage models have been introduced in this context. However, it is often reported that a considerable range of geomaterials exhibit two dissipative mechanisms.

The experimental results from triaxial tests performed by Chiarelli (2000) have shown two basic phenomena on the overall mechanical responses of the sedimentary rocks (Chiarelli et al., 2003). Irreversible strains essentially related to plastic deformation by clay sheet sliding which is associated with a progressive damage (Alba et al. 2009; Zheng et al. 2014). Accordingly both of these dissipative aspects should be taken into account; therefore an elastoplastic damage model is required for constitutive modelling of such geomaterials.

For constitutive modelling of multi-phase unsaturated porous medium, complex aspects of governing mechanisms should be adequately accounted for. In such media, mechanical response (plastic flow and fracturing) combined with suction (capillary) effects and water retention characteristics should be taken into consideration, which undoubtedly induce complex poromechanical couplings. The model dedicated to this class of materials should be capable of addressing such effects in a sufficiently rigorous manner. The adoption of stress state variables is the first step and an essential prerequisite for a suitable formulation of such models. Hence, a chronological development of stress variables for modelling of unsaturated porous media is briefly reviewed in the sequel to summarize the principal ideas (e.g. independent and combined stresses) to adopt most appropriate ones.

The difficulty was early realized in 1950s and 1960. During this period, concentrated researches were carried out to investigate unsaturated soils behaviour and a large effort was made to interpret the experimental observations by use of extension of Terzaghi's Effective stress to the unsaturated state. Bishop's effective stress (Bishop, 1959) was one of the well-known definitions which clearly failed to justify unsaturated soils behaviour, particularly, in the case of volumetric behaviour.

Development of effective stress expressions for unsaturated soils, therefore, came inevitably under criticism and the notion of adoption of two stress variables emerged in the geotechnical literature (e.g. Coleman, 1962; Bishop and Blight, 
1963; Blight, 1967). Matyas and Radhakrishna (1968) followed this framework and proposed their state surfaces for degree of saturation and void ratio. The state surfaces were originally introduced to explain the hydro-mechanical behaviour of unsaturated soils and are typically accompanied with nonlinear elasticity problems (Gatmiri \& Delage, 1997; Gatmiri et al., 1997).

Fredlund \& Morgenstern (1977) proposed a set of stress state variables for unsaturated soils. They presented the results of null tests supporting their choice and indicated that any couple of variables among $\left(\boldsymbol{\sigma}-u_{a}\right),\left(\boldsymbol{\sigma}-u_{w}\right)$ and $\left(u_{a}-u_{w}\right)$ can be employed as stress state variables for modelling of unsaturated soils behaviour.

Alonso et al. (1990) pioneered elastoplastic constitutive modelling of unsaturated soils by their celebrated model which was later referred as Barcelona Basic Model (BBM). BBM uses two independent stress variables, net stress (the total stress in excess of pore air pressure) and the suction (the pore air pressure in excess of pore water pressure), as a basis for its formulation.

In recent decades there has been a renewal of interest in justification and application of pseudo-effective stress definitions for unsaturated porous media. For instance, Khalili and Khabbaz (1998) demonstrated supporting evidence for the validity of Bishop's effective stress expression. They proposed a relationship between the effective stress parameter $\chi$ and the suction ratio (ratio of suction over the air entry value). Some authors (e.g. Hutter et al., 1999) made attempts to validate Bishop's effective stress by making use of averaging methods and mixture theories. Sheng et al. (2003a) used the term 'Constitutive stress' for the equation (1) as a state variable:

$$
\boldsymbol{\sigma}^{\prime}=\boldsymbol{\sigma}-\mathbf{m} \varphi\left(S_{r}\right) u_{w}
$$

where $\mathbf{m}$ is the column vector with 1 at normal stress entries and 0 at shear stress entries, $\varphi\left(S_{r}\right)$ is the constitutive stress parameter that depends on the degree of saturation $S_{r}$ or the suction, and $u_{w}$ is the pore water pressure. To eliminate the need for a material parameter for the constitutive stress function, this function is set to either $\varphi=S_{r}$ or $\varphi=\sqrt{S_{r}}$ in the problems analyzed by Sheng et al. (2003b).

The resurgence of the models based on Bishop-type effective stress in recent years is accompanied with realization of the fact that two stress variables are required for modelling of an unsaturated soil medium. Indeed, as Jommi (2000) pointed out "in fact, no single stress variable has ever been found which, substituted for effective stress, allows for a description of all the aspects of the mechanical behaviour of a given soil in the unsaturated range." As a result in such models, the suction is used as a hardening variable or a stress variable along with the other variable. Accordingly, Laloui and Nuth (2008) and Nuth and Laloui (2008) proposed the following set of stresses for the modelling of 
unsaturated soils and referred to them as the 'generalised stresses' for unsaturated soils in which Bishop's stress and suction are respectively conjugated to strain and degree of saturation.

$$
\begin{gathered}
\boldsymbol{\sigma}_{\text {Bishop }}^{\prime} \leftrightarrow \boldsymbol{\varepsilon} \\
s \leftrightarrow S_{r}
\end{gathered}
$$

Overall, the complex stress variables (e.g. Bishop's effective stress) are dependent on material states and therefore are not straightforwardly controllable in conventional laboratory testing procedures. It is not feasible to develop an entirely new constitutive relationship in terms of these variables, unless an existing framework is used (Sheng et al., 2008). In the models which make use of the Bishop's effective stress with the parameter $\chi=S_{r}$, the inherent hydromechanical coupling is greatly dependent on the water retention relation used in the model. This entails utilizing sophisticated water retention models. Consequently the water retention properties of unsaturated medium should be specifically determined and it may necessitate the use of hysteretic water retention models in addition to mechanical formulation.

Tarantino and Mongiovi (2000) and Tarantino et al. (2000) carried out some tests which strongly support the proposition of independent stress state variables by Fredlund and Morgenstern (1977). This would be due to the fact that independent stress state variables can be considered as reflection of the most meaningful physical quantities of an unsaturated medium. In fact, preference of these variables is based upon the supporting experiments. Experimental observations made to date have confirmed the physical appropriateness of the adoption of independent stress variables for the modelling of governing mechanisms of unsaturated media. As cited by Fredlund (2006) “... it is the two independent stress tensors containing stress state variables (i.e., [net stress] $\boldsymbol{\sigma}-u_{a}$ and matric suction, $u_{a}-u_{w}$ ) that form the most general and fundamental basis for the development of a science for unsaturated soil mechanics."

On the contrary, some researchers believe that the choice between two independent stress variables or a single effective stress (coupled with retention curve) remains a matter of preference and convenience.

Considering the above, independent stress state variables including suction and net stress are adopted as stress variables of the present model.

Different aspects of the modelling of stress-strain and volume change behaviour of unsaturated media with inclusion of thermal effects are comprehensively presented and discussed in Ahangar-Asr et al. 2015.

In this paper, a new model is formulated within the context of plasticity and continuum damage mechanics. The new poro-plasto-damage model involves damage and thermal dependency of plastic yield surface. In order to characterize 
the influence of fracturing on fluid transfers, damage variable is also included in the expression of the intrinsic liquid permeability of the medium. The model has been implemented in $\Theta$-STOCK Code which is a finite element code for analysis of coupled problems of multiphase porous media. The program is developed by Gatmiri and his co-workers and validated by various research and practical problems of geotechnical engineering (Alizadeh and Gatmiri, 2015a\&b; Arson and Gatmiri, 2012; Arson and Gatmiri, 2011; Gatmiri et al., 2010a; Gatmiri and Arson, 2008; Gatmiri and Delage, 1997).

Following previous models of Gatmiri (Gatmiri, 2002; Gatmiri and Hoor, 2007; Gatmiri et al., 2010b), a fully coupled thermo-hydro-mechanical formulation is employed for the fluid flows and the balance equations of the model. The proposed model is expected to be a useful tool for simulation of complex, coupled response of engineered and geological barriers surrounding heat-emitting waste canisters in the context of geological disposal of high-level nuclear wastes.

\section{Conceptualization of semi-brittle behaviour}

Argillaceous formations are considered as a possible host rock for high level radio- active nuclear waste repository (Dehandschutter et al. 2004; Gaudin et al. 2009; Wang et al. 2014). One of these clay stones is the Callovo-Oxfordian (COx) argillaceous formation (Montes et al. 2004; Zhang et al. 2012). This argillite is a complex material composed of different minerals. It would be described as a clayey matrix with embedded calcite and quartz particles and several other minerals in lower content. Some quartz grains are partially rounded while carbonate particles are both shell fragments and idiomorphic calcite crystals. Such a mineralogical structure leads to a complex behaviour at various scales when the material is subjected to loading. High calcite content may increase the overall rigidity of the material making it more brittle and stiffer while a high argillaceous content leads to a more ductile behaviour and increased plasticity which may induce larger plastic strains in medium (Chiarelli et al. 2000; Elie et al. 2004; Magnenet et al. 2011).

Chiarelli 2000 carried out some tests on the samples from this argillite and outlined its behavioural features. Some of his results are shown in figure 1. One can observe that two behavioural characteristics are paramount in overall response of this clay stone. Each unloading cycle is associated with large residual strains as well as progressive degradation of the stiffness.

The plastic deformation is mainly attributed to the sliding of clay sheets though the twilling of big calcite grains can indeed play a part in this phenomenon. The second mechanism is anisotropic damage which is induced by oriented microcracks. These micro-cracks which are mainly concentrated at the interfaces between grains and argillaceous matrix degrade the material properties between 
each unloading reloading cycle. There is an obvious correlation between tensile strains and induced damage, which follows an anisotropic pattern.

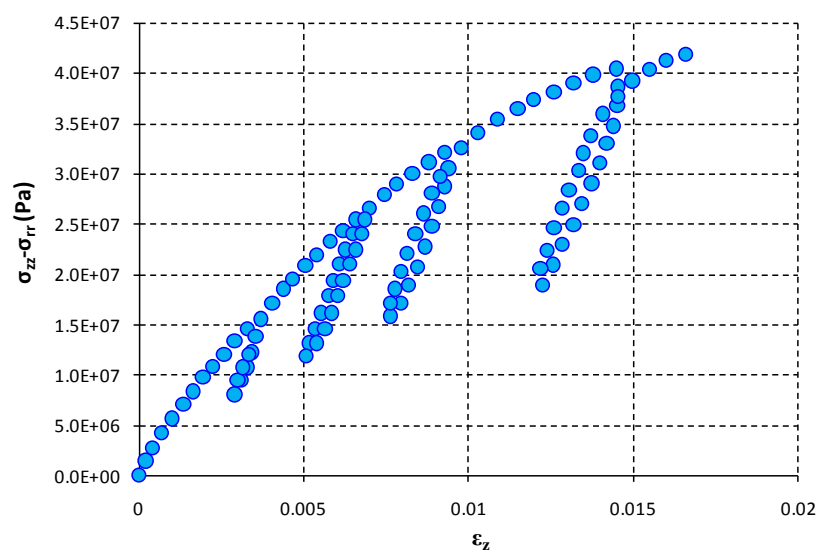

Figure 1: Stress-strain curve for argillite with unloading-reloading cycles (after Chiarelli, 2000)

In view of these results, a properly formulated elastoplastic damage model provides the best alternative for constitutive modelling of such semi-brittle geomaterials. A number of elastoplastic damage models to date have been suggested; however most of them are based on simplifying assumptions like the use of Bishop's like effective stress or isotropic damage variable.

As it was mentioned in introductory section, independent stress state variables provide the most meaningful physical quantities of an unsaturated medium and experimental results support this proposition. Accordingly this paper expounds a new plasto-damage model employing independent stress state variables as well as an anisotropic definition of damage which can appropriately accommodate behavioural patterns of such semi-brittle geomaterials.

\section{Characterization of anisotropic damaging process}

\subsection{Damaged effective stress variables}

The term 'effective stress' used in damage mechanics should be clearly distinguished from the Terzaghi's effective stress concept in soil mechanics. The so-called effective stress concept has been commonly used in continuum damage mechanics since early introduction by Kachanov (1958). In this framework, for a single phase solid medium, Cauchy stress tensor is replaced by the effective stress tensor and this approach employs the constitutive laws of effective (undamaged) material to model whole damaged medium. 
Figure 2 illustrates the physical basis for the definition of damage-related effective stress at meso-scale. A Representative Elementary Volume (REV) of a single phase medium is considered which is damaged by an arbitrary distribution of micro-cracks with different orientations. The REV is submitted to a uniform stress field on its boundaries. Neglecting the gravitational body force, the externally applied stresses can be regarded as the nominal stresses within the REV.

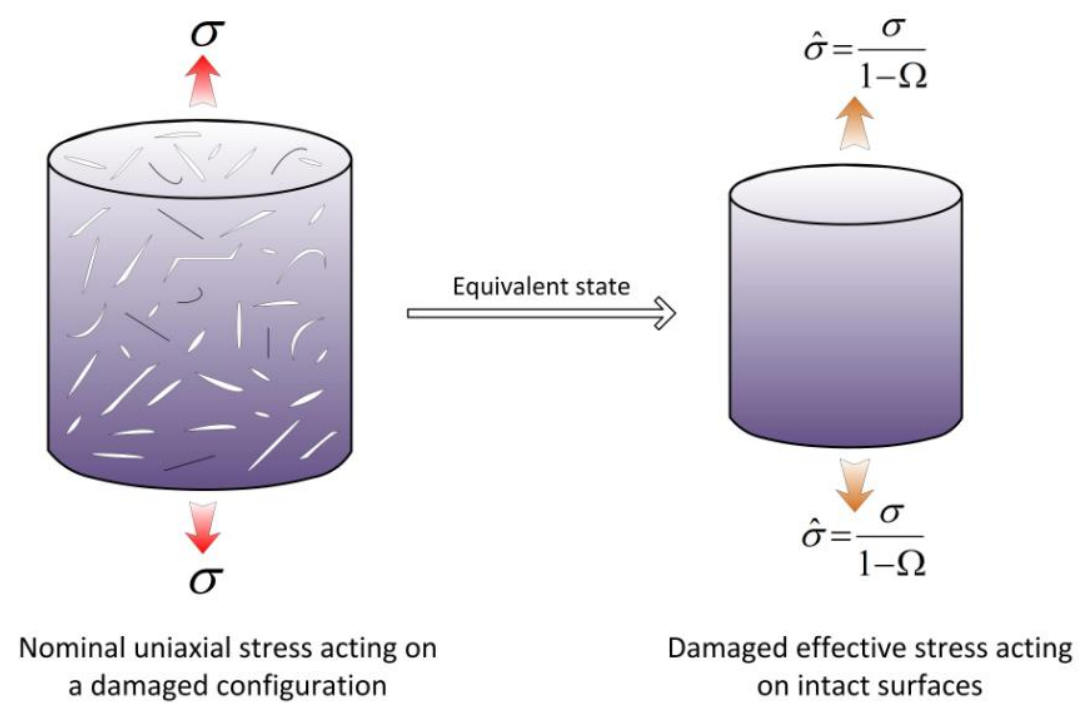

Figure 2: Representation of the effective damaged stress for a cylindrical REV under uniaxial tension.

The total reduction of effective material area is shown by 'Damaged Area' which indicates the area of micro-defects in each direction. With the assumption of isotropic damage propagation, this loss of effective area is equal for all directions of the medium. Based on the reduction of the effective area, isotropic damage variable can be defined as follows:

$\Omega=\frac{\text { Total Area }- \text { Damaged Area }}{\text { Total Area }}$

Taking account of the loss of the load carrying capacity of the REV, the damaged effective stress can be written as follows:

$$
\hat{\boldsymbol{\sigma}}=\frac{\boldsymbol{\sigma}}{1-\Omega}
$$


This effective stress is here referred to as the 'damaged effective stress variable'. At this stage, the damaged effective stress variables should be adopted for the unsaturated state. An equation similar to 5 is classically employed for definition of damaged effective stress in the case of single phase damaged media. Where $\mathbf{M}$ is a fourth-order operator relating the damaged effective stresses to Cauchy's stress tensor:

$$
\underline{\underline{\hat{\boldsymbol{\sigma}}}}=\underline{\underline{\underline{\underline{\mathbf{M}}}}}: \underline{\underline{\boldsymbol{\sigma}}}
$$

Numerous mathematical forms have been proposed for the operator $\mathbf{M}$ in the CDM context. The operators which ensure symmetrisation of the damaged effective stress are preferred. Hence, in order to transform independent stress variables of model to the damaged state, the following expressions are employed.

$$
\begin{aligned}
& \underline{\underline{\hat{\boldsymbol{\sigma}}^{\prime \prime}}}=(\underline{\underline{\underline{\mathbf{I}}}}-\underline{\underline{\boldsymbol{\Omega}}})^{-1 / 2} \cdot \underline{\underline{\boldsymbol{\sigma}^{\prime \prime}}} \cdot(\underline{\underline{\underline{\mathbf{I}}}}-\underline{\underline{\boldsymbol{\Omega}}})^{-1 / 2} \\
& \hat{s}=\frac{s}{3}(\underline{\underline{\underline{\mathbf{I}}}}-\underline{\underline{\boldsymbol{\Omega}}})^{-1}: \underline{\underline{\mathbf{I}}}
\end{aligned}
$$

In fact, the operator of Cordebois and Sidoroff (1982) is used for definition of damaged net stress, $\underline{\underline{\underline{\hat{\sigma}^{\prime \prime}}}}$, and damaged suction, $\hat{s}$. These variables can facilitates transformation between virgin state of medium and damaged configuration. This transformation should be carried out by use of either the Principle of equivalent elastic energy or Principle of strain equivalence. In the current study the strain equivalence hypothesis is adopted, which basically states that the strain associated with a damaged state under the applied stress is equivalent to the strain associated with its undamaged state under the effective stress (Hansen and Schreyer, 1994). This assumption is widely used by other authors (e.g. Ju, 1989). Following the strain equivalence hypothesis, the damaged medium behaviour is formulated in the damaged effective stress and the actual strain space. Now an appropriate damage variable needs to be defined to represent an anisotropic damaging process.

\subsection{Damage variable}

Damage variable is a macroscopic measure of microscopic degradation of a Representative Elementary Volume (Voyiadjis and Deliktas, 2000). Damage in materials can be represented in many forms such as specific void and crack surfaces, specific crack and void volumes, the spacing between cracks or voids, scalar illustration of damage, and general tensorial representation of damage (Abu Al-Rub and Voyiadjis, 2003).

Kachanov (1992) demonstrated that a second order tensorial damage variable can rigorously portray the microcracks distribution in a two-dimensional configuration, and it can also present a good approximation in the case of three- 
dimensional problems. Therefore the second order variable $\underline{\underline{\boldsymbol{\Omega}}}$ is adopted as damage variable at the mesoscopic scale:

$$
\underline{\underline{\mathbf{\Omega}}}=\sum_{i=1}^{3} d_{i} \underline{n_{i}} \otimes \underline{n_{i}}
$$

This definition is based on spectral decomposition of Ortiz (1985). As it is illustrated for a two dimensional case in Fig. 3, the approximately parallel microdefects and micro-cracks developed in the Representative Elementary Volume (REV) is considered as orthogonal equivalent meso-cracks.
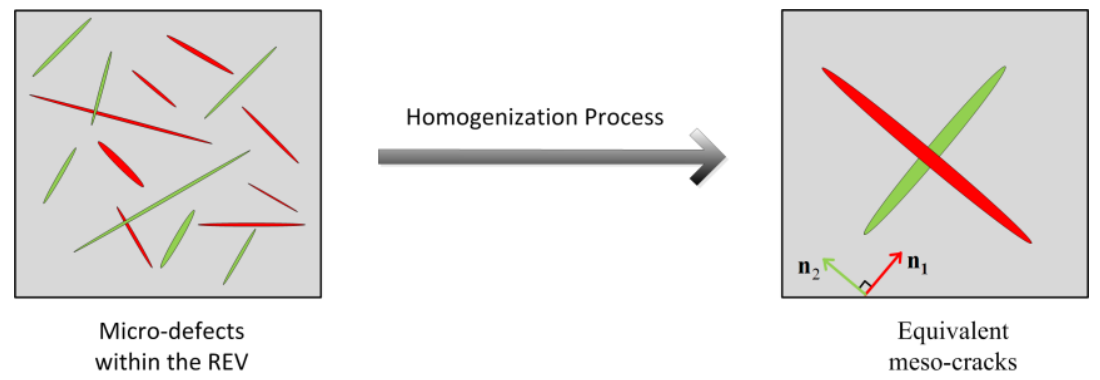

Figure 3: Fictitious representation of homogenization process of the microdefects within a two dimensional REV.

The fictive equivalent meso-cracks are characterized by unit normal vector of fracturing plane, $\underline{n_{i}}$, and crack density parameter $d_{i}$. The intensity of damage within the control volume can be described by the volume of equivalent mesocracks divided by the volume of REV. assuming penny-shaped meso-cracks with a radius of $R_{i}$ and an opening of $e_{i}$, crack density parameter can be written as follows:

$d_{i}=\frac{\pi\left(R_{i}\right)^{2}\left(e_{i}\right)}{V_{R E V}}$

\subsection{Damage Criterion and evolution law}

According to the experimental results reported in literature, there is an obvious correlation between total tensile strains and induced damage in brittle geomaterials. We can, therefore, assume that damage growth is associated with the development of generalized tensile strain and damage start developing within the medium as the strains reach a threshold. Accordingly the equation 9 is used as damage yield function.

$$
F_{d}\left(\boldsymbol{\varepsilon}^{+}, \mathbf{\Omega}\right)=\sqrt{\frac{1}{2} g \boldsymbol{\varepsilon}^{+}: g \boldsymbol{\varepsilon}^{+}}-C_{0}-C_{1}(\mathbf{I d}: \mathbf{\Omega})
$$


$C_{0}$ and $C_{1}$ are material parameters wherein $C_{0}$ designates the initial damage threshold, and $C_{1}$ characterizes the degradation of the medium materials with cumulated damage. This means that under multiaxial stress states, the REV still continues damaging in tensile mode when the combination of major tensile strains attain damage driving threshold. It should be noted that the term $g \boldsymbol{\varepsilon}^{+}$is part of damage-conjugated stress or 'damage affinity' that the product of it by damage is an energy release rate.

Assuming the sign convention of solid mechanics (compression negative), the positive part of total strain, $\boldsymbol{\varepsilon}^{+}$, represents the extensional components of deformations. Thus the spectral decomposition of total strains (Ortiz, 1985; Ju, 1989) can be used:

$\boldsymbol{\varepsilon}^{+}=\sum_{k=1}^{3} \varepsilon_{k} H\left(\varepsilon_{k}\right) \mathbf{W}_{k} \otimes \mathbf{W}_{k}$

where $\varepsilon_{k}$ is $\mathrm{k}^{\text {th }}$ eigenvalue of the strain tensor which corresponds to $\mathrm{k}^{\text {th }}$ principal strain, $\mathbf{W}_{k}$ is eigenvector (principal vector) associated to $\varepsilon_{k}$ and $H\left(\varepsilon_{k}\right)$ is the Heaviside function of the principal strain, $H\left(\varepsilon_{k}\right)=1$ for $\varepsilon_{k} \geq 0, H\left(\varepsilon_{k}\right)=0$ for $\varepsilon_{k}<0$. Assuming a normal dissipation scheme for the damage, damage evolution law can be written as follows:

$\dot{\boldsymbol{\Omega}}=\dot{\Lambda}_{d} \frac{\partial F_{d}(\boldsymbol{\varepsilon}, \boldsymbol{\Omega})}{\partial \boldsymbol{\varepsilon}^{+}}=\dot{\Lambda}_{d} \frac{\boldsymbol{\varepsilon}^{+}}{\sqrt{2 \operatorname{tr}\left(\boldsymbol{\varepsilon}^{+} \boldsymbol{\varepsilon}^{+}\right)}}$

This treatment can guarantee that, the evolution rate of damage variable and the tensor of tensile strains are developing in the same direction. The evolution of positive scalar damage multiplier, $\dot{\Lambda}_{d}$, can be determined by consistency condition of the damage criterion.

\section{Characterization of elastoplastic behaviour}

\subsection{Elastic formulation}

First, the formulation of elastic behavior should be presented. To guarantee that an elasticity formulation is thermodynamically acceptable the elastic behaviour laws must be derived from a free energy potential or alternatively from a complementary energy potential in the framework of hyperelasticity Houlsby et al. (2005). Thus we extended a potential introduced by Hueckel and Borsetto (1990) to the unsaturated state as follows: 


$$
\begin{aligned}
& G(p, s, T)=\left(\alpha_{0}^{*}+\alpha_{2} \Delta T\right) \Delta T\left(p-p_{g e o}\right)+\left(\frac{\kappa_{i}}{1+e_{0}}+\alpha_{1} \Delta T+\alpha_{3} \Delta T^{2}\right) p_{g e o}\left[\frac{p}{p_{g e o}}\left(\ln \frac{p}{p_{g e o}}-1\right)+1\right] \\
& +\left(p-p_{g e o}\right) \frac{\kappa_{s}}{1+e_{0}} \ln \left(\frac{s+p_{\text {atm }}}{s_{\text {geo }}+p_{\text {atm }}}\right)
\end{aligned}
$$

Where $\kappa_{i}$ is isothermal elastic compressibility (or the conventional unloadingreloading compression index), $e_{0}$ is initial void ratio, $p_{g e o}$ is isotropic component of reference stress or geostatic stress. $\alpha_{0}^{*}, \alpha_{1}, \alpha_{2}, \alpha_{3}$ are model constants. Hence the isotropic volumetric strain can be written as follows:

$d \varepsilon_{v}^{e}=\left(\frac{\kappa_{i}}{1+e_{0}}+\left(\alpha_{1}+\alpha_{3} \Delta T\right) \Delta T\right) \frac{d p}{p}+\left(\frac{\kappa_{s}}{1+e_{0}} \frac{1}{s+p_{a t m}}\right) d s+\left[\left(\alpha_{0}^{*}+2 \alpha_{2} \Delta T\right)+\left(\alpha_{1}+2 \alpha_{3} \Delta T\right) \ln \frac{p}{p_{\text {geo }}}\right] d T$

Now the expression for thermal elastic compressibility index $\kappa_{T}$ can be obtained as follows:

$$
\kappa_{T}=\left[\frac{\kappa_{i}}{1+e_{0}}+\left(\alpha_{1}+\alpha_{3} \Delta T\right) \Delta T\right]\left(1+e_{0}\right)
$$

\subsection{Degradation of elastic properties}

Degradation of elastic properties is one of main features of mechanical behavior of argillites which has often been attributed to the induced damage by microcracks. Different forms of relations are introduced in the literature to reproduce such effect. Equation (12) is employed here to modify the bulk modulus and shear modulus of undamaged material undergoing damage propagation. Non-interacting microcracks and moderate damage assumption are made to obtain these relations (Budiansky and O'Connell 1976; Shao et al. 2006)

$$
B(\boldsymbol{\Omega})=B_{0}\left(1-\frac{1}{3} \beta_{1} \operatorname{tr} \mathbf{\Omega}\right) \quad G(\mathbf{\Omega})=G_{0}\left(1-\frac{1}{3} \beta_{2} \operatorname{tr} \mathbf{\Omega}\right)
$$

where $\beta_{1}$ and $\beta_{2}$ are material parameters characterizing the damage effects on the elastic properties of the geomaterial.

\subsection{Plastic behaviour}

The following equation is adopted as plastic yield surface for the present study:

$F_{p}=\hat{q}^{2}-M^{2}\left(\hat{p}+\hat{p}_{s}\right)\left(\hat{p}_{0}-\hat{p}\right)=0$

with: 


$$
\begin{aligned}
& p_{s}=p_{s}(\hat{s}, T, \boldsymbol{\Omega})=k \cdot \hat{s} .(1-\alpha T) \\
& p_{c}=p_{c 0} \cdot \exp \left(\gamma_{H} \varepsilon_{v}^{p}\right)\left[1-\gamma_{T} \log \left(\frac{T}{T_{0}}\right)\right] \\
& \hat{p}_{s}=p_{s}\left(1+\frac{1}{3} \xi \operatorname{tr} \boldsymbol{\Omega}\right) \\
& \hat{p}_{c}=p_{c}\left(1+\frac{1}{3} \xi \operatorname{tr} \boldsymbol{\Omega}\right) \\
& \hat{p}_{0}=\hat{p}_{0}\left(\hat{s}, \varepsilon_{v}^{p}, T, \boldsymbol{\Omega}\right)=p_{r e f}\left(\frac{\hat{p}_{c}}{p_{r e f}}\right)^{\frac{\lambda(0)-\kappa_{T}}{\lambda(\hat{s})-\kappa_{T}}}
\end{aligned}
$$

$\hat{p}_{c}$ is the apparent preconsolidation stress for the saturated state and $\hat{p}_{0}$ is its counterpart for the unsaturated state. $\hat{p}_{s}$ represents the variation of cohesion with the corresponding suction or damage changes. $k, \xi, \gamma_{H}, \gamma_{T}$ are material parameters through which the above mentioned variables are defined. $p_{\text {ref }}$ is the reference stress for the plastic yield surface.

As discussed earlier, non-isothermal conditions prevail in the vicinity of underground nuclear waste repositories, consequently in this paper Barcelona Basic Model is extended to a non-isothermal formulation. To this end, apparent preconsolidation stress $p_{c}$ is considered to be dependent on the damaged suction and accumulated thermoplastic strains as well as on the temperature. $p_{s}$ also depends on temperature and damaged suction. On the other hand, in order to account for the influence of damage on plastic yield locus, $p_{c}$ and $p_{s}$ are transformed into the damaged state. In fact, $\hat{p}_{c}$ and $\hat{p}_{s}$ are damaged counterparts of $p_{c}$ and $p_{s}$.

This formulation results in shrinkage of the elastic domain due to temperature increase. Experimental results available in the literature support the assumption that the region of elastic behaviour, that is, the extent of a yield locus, decreases with heating.

It is evident that the yield function is written based on the damaged stress components $\hat{q}, \hat{p}$. In fact, the damaged effective stress variables play the role of coupling variables for the current elastoplastic damage model which can readily couple the damage influence with the elastic criterion and the plastic formulation. The term $\left(1+\frac{1}{3} \xi \operatorname{tr} \Omega\right)$ is multiplied by the undamaged variables $p_{s}$ and $p_{c}$ to reflect variation of plastic yield locus with damage.

Fig. 4 illustrates the yield loci of the proposed model for the undamaged and damaged states. The model is referred to as Damage Barcelona Basic Model $(\mathrm{DBBM})$ in the following. As can be seen, damage can shrink or swell the elastic 
domain. Adoption of positive value for $\xi$ can enlarge the elastic domain while a negative value of $\xi$ has an opposite effect resulting in shrinkage of the extent of the yield locus with increase of damage intensity.

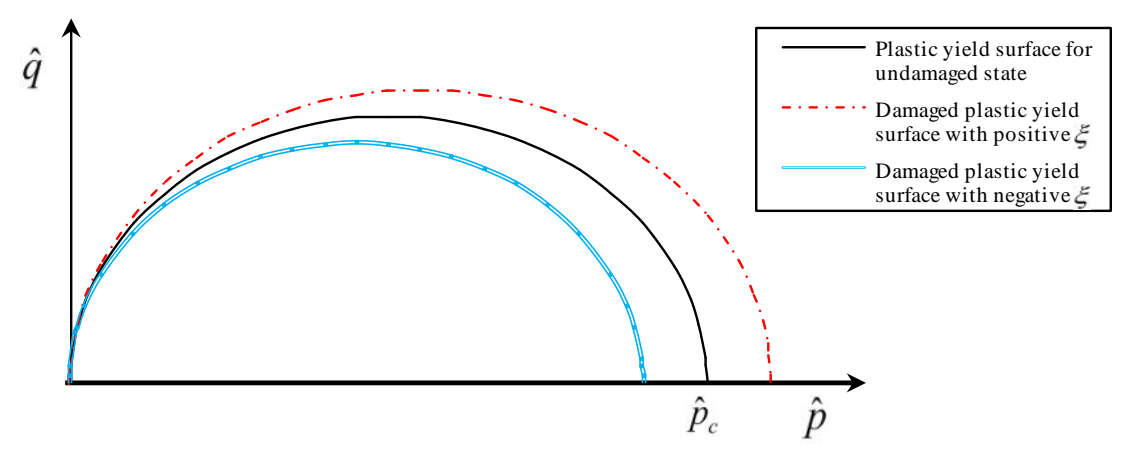

Figure 4: The yield surface of Elasto-plastic damage model (DBBM)

A suction-dependent relation for virgin compressibility index is adopted which is the same as that used by Alonso et al. (1990):

$$
\lambda(s)=\lambda(0)[(1-r) \exp (-\beta \hat{s})+r]
$$

where $r$ is a parameter controlling the maximum stiffness of the porous medium for an infinite suction, $r=\lambda(\hat{s} \rightarrow \infty) / \lambda(0)$, and $\beta$ is a parameter which characterizes the rate of increase of soil stiffness with suction.

Under small strain condition, total strain can be decomposed into an elastic part and a plastic part. Then, under the assumption of associated flow-rule, increment of plastic strain can be written as follows:

$d \boldsymbol{\varepsilon}^{p}=d \Lambda_{p} \frac{\partial F_{p}}{\partial \boldsymbol{\sigma}}$

Application of consistency condition to the plastic yield surface leads to the following expression for plastic multiplier:

$$
d \Lambda_{p}=\frac{\left(\frac{\partial F_{p}}{\partial \boldsymbol{\sigma}}\right) \mathbf{D}^{e} d \boldsymbol{\varepsilon}+\left(\frac{\partial F_{p}}{\partial s}-\frac{\partial F_{p}}{\partial \boldsymbol{\sigma}} \mathbf{D}_{e s}\right) d s+\left(\frac{\partial F_{p}}{\partial T}-\frac{\partial F_{p}}{\partial \boldsymbol{\sigma}} \mathbf{D}_{e T}\right) d T-\left(\frac{\partial F_{p}}{\partial \boldsymbol{\Omega}}\right) d \boldsymbol{\Omega}}{\left(\frac{\partial F_{p}}{\partial \boldsymbol{\sigma}}\right)^{T} \mathbf{D}^{e} \frac{\partial F_{p}}{\partial \boldsymbol{\sigma}}-\frac{\partial F_{p}}{\partial \varepsilon_{v}^{p}} \frac{\partial F_{p}}{\partial p}}
$$

Finally, incremental constitutive equation can be obtained as follows:

$$
d \boldsymbol{\sigma}=\left(\mathbf{D}^{e}-\mathbf{D}^{p}\right) d \boldsymbol{\varepsilon}-\left(\mathbf{D}_{e T}+\mathbf{D}_{e T}^{p}-\mathbf{D}_{e T e}^{p}\right) d T-\left(\mathbf{D}_{e s}+\mathbf{D}_{e s}^{p}-\mathbf{D}_{e s e}^{p}\right) d s-\left(\mathbf{D}_{\Omega}\right) d \boldsymbol{\Omega}
$$


In which the following terms are used:

$$
\begin{aligned}
& \mathbf{D}_{s}=\beta_{s} \mathbf{m}^{T}=\left(\frac{\kappa_{s}}{1+e_{0}} \frac{1}{s+p_{a t m}}\right) \mathbf{m}^{T} \\
& \beta_{T}=\left(\alpha_{0}^{*}+2 \alpha_{2} \Delta T\right)+\left(\alpha_{1}+2 \alpha_{3} \Delta T\right) \ln \frac{p}{p_{\text {geo }}} \\
& \mathbf{D}_{T}=\beta_{T} \mathbf{m}^{T} \\
& \mathbf{a}=\frac{\partial F_{p}}{\partial \boldsymbol{\sigma}} \\
& b=\frac{\partial F_{p}}{\partial s} \\
& c=\frac{\partial F_{p}}{\partial T} \\
& \mathbf{d}=\frac{\partial F_{p}}{\partial \boldsymbol{\Omega}} \\
& \mathbf{D}^{p}=\frac{\left(\mathbf{a}^{T} \mathbf{D}^{e}\right)\left(\mathbf{D}^{e} \mathbf{a}\right)}{H_{1}} \\
& \mathbf{D}_{e s}=\mathbf{D}^{e} \mathbf{D}_{s}^{-1} \\
& \mathbf{D}_{e T}=\mathbf{D}^{e} \mathbf{D}_{T}^{-1} \\
& \mathbf{D}_{e s}^{p}=\frac{b \mathbf{D}^{e} \mathbf{a}}{H_{1}} \\
& \mathbf{D}_{e T}^{p}=\frac{c \mathbf{D}^{e} \mathbf{a}}{H_{1}} \\
& \mathbf{D}_{e s e}^{p}=\frac{\mathbf{a}^{T} \mathbf{D}^{e} \mathbf{D}_{s}^{-1}\left(\mathbf{D}^{e} \mathbf{a}\right)}{H_{1}} \\
& \mathbf{D}_{e T e}^{p}=\frac{\mathbf{a}^{T} \mathbf{D}^{e} \mathbf{D}_{T}^{-1}\left(\mathbf{D}^{e} \mathbf{a}\right)}{H_{1}} \\
& \mathbf{D}_{\Omega}=\frac{\left(\mathbf{d D}^{e} \mathbf{a}\right)}{H_{1}} \\
& H_{1}=H-H_{c r}=\mathbf{a}^{T} \mathbf{D}^{e} \mathbf{a}-\frac{\partial F_{p}}{\partial \varepsilon_{v}^{p}} \frac{\partial F_{p}}{\partial p}
\end{aligned}
$$

It is evident that the classical hardening plasticity is followed for the plastic flow modelling in this context although other approaches like generalized plasticity 
would be applicable for this framework. Furthermore other approaches like Ahangar-Asr et al. 2015 would be appropriate for the modelling of thermomechanical behaviour of unsaturated media.

\section{Transfer and balance equations}

For the transfer and balance equations in porous media several formulations have been described in the literature. Following previous models of Gatmiri (Gatmiri et al., 1998; Gatmiri, 2002; Gatmiri and Hoor, 2007; Jabbari and Gatmiri 2007; Gatmiri et al., 2010a,b; Arson and Gatmiri, 2012), a coupled formulation is employed for the fluid flows and the balance equations. The formulation used in the model is outlined in the following.

Total moisture transfer in an unsaturated porous medium submitted to a temperature gradient is the superposition of the flows that take place separately in each phase of vapour and liquid. Therefore, the governing equation for total moisture movement is defined as the sum of liquid and vapour velocities:

$$
\mathbf{U}+\mathbf{V}_{\text {vap }}=\frac{\mathbf{q}_{\mathrm{W}}}{\rho_{w}}+\frac{\mathbf{q}_{\text {vap }}}{\rho_{w}}=\left[-\mathbf{D}_{\text {TW }} \nabla(T)-\mathbf{D}_{\text {SW }} \nabla(s)-\mathbf{D}_{\mathbf{w}} \nabla(z)\right]+\left[-\mathbf{D}_{\mathrm{TV}} \nabla(T)-\mathbf{D}_{\mathrm{SV}} \nabla(s)\right]
$$

where $\mathrm{z}$ is elevation for representation of gravitational potential. The above equation can be rewritten as follows:

$$
\frac{\mathbf{q}}{\rho_{w}}=-\mathbf{D}_{\mathbf{T}} \nabla(T)-\mathbf{D}_{\mathbf{S}} \nabla(s)-\mathbf{D}_{\mathbf{W}} \nabla(z)
$$

Where $\mathbf{D}_{\mathbf{T}}$ is the thermal moisture diffusivity, which is equal to the sum of the thermal vapour and water diffusivities. $\mathbf{D}_{\mathbf{s}}$ is the isothermal moisture diffusivity, which is equal to the sum of the isothermal vapour and water diffusivities. The last term is the gravitational part of the equation and $\mathbf{D}_{\mathrm{w}}$ can be defined as:

$$
\mathbf{D}_{\mathbf{w}}=\mathbf{K}_{\mathbf{w}}\left(n, \boldsymbol{\Omega}, S_{w}, T\right)=K_{r t}(T) K_{r c}\left(S_{w}\right) \mathbf{K}_{\text {int }}^{\text {tot }}(n, \boldsymbol{\Omega})
$$

In which $K_{r t}(T), K_{r c}\left(S_{w}\right), \mathbf{K}_{\text {int }}^{\text {tot }}(n, \boldsymbol{\Omega})$ are, respectively, relative thermal, capillary and intrinsic permeability of the medium:

$$
\begin{aligned}
& K_{r t}(T)=\frac{\eta_{w}(T)}{\eta_{w}\left(T_{r e f}\right)} \\
& K_{r c}\left(S_{w}\right)=\left(\frac{S_{w}-S_{w, r}}{1-S_{w, r}}\right)^{d}, \quad 3<d<4
\end{aligned}
$$




$$
\mathbf{K}_{\text {int }}^{\text {tot }}\left(n^{r e v}, n^{i r r}, \mathbf{\Omega}\right)=\mathbf{K}_{\text {int }}^{\text {und }}\left(n^{r e v}\right)+\mathbf{K}_{\text {int }}^{\mathbf{d a m}}\left(n^{i r r}, \mathbf{\Omega}\right)
$$

As can be seen the intrinsic permeability tensor is split into a part for intact medium and a damage-related part:

$$
\mathbf{K}_{\text {int }}^{\mathbf{u n d}}(n)=k_{w_{0}} 10^{\alpha_{w} e} \mathbf{I d}
$$

For the moisture mass conservation, the following equation is used:

$$
\frac{\partial \rho_{\text {mois }}}{\partial t}=\frac{\partial\left(\theta_{w} \rho_{w}+\left(n-\theta_{w}\right) \rho_{\text {vap }}\right)}{\partial t}=\frac{\partial\left(n S_{w} \rho_{w}+n\left(1-S_{w}\right) \rho_{\text {vap }}\right)}{\partial t}-\operatorname{div}\left(\rho_{w}\left(\mathbf{U}+\mathbf{V}_{\text {vap }}\right)\right)
$$

Assuming that gas flow is governed by Darcy's law, one can write the generalised form as follows:

$$
\mathbf{V}_{\mathbf{a}}=-\mathbf{K}_{\mathbf{a}} \cdot \nabla\left(\frac{p_{\text {air }}}{\gamma_{\text {air }}}+z\right)
$$

For a non isothermal unsaturated porous medium, air permeability can be expressed as:

$$
\mathbf{K}_{\mathrm{a}}=C_{a} \frac{\gamma_{\text {air }}}{\eta_{\text {air }}}\left[e\left(1-S_{w}\right)\right]^{\alpha_{a}} \mathbf{I d}
$$

The governing differential equation for the mass conservation of the gas phase can be expressed, as follows:

$$
\frac{\partial}{\partial t}\left[\rho_{a}\left(n-\theta_{w}+H \theta_{w}\right)\right]=-\operatorname{div}\left(\rho_{a} \mathbf{V}_{\mathbf{a}}\right)-\operatorname{div}\left(\rho_{a} H \mathbf{U}\right)+\rho_{W} \operatorname{div}\left(\mathbf{V}_{\mathbf{v a p}}\right)
$$

where $H$ is Henry's coefficient and denotes the amount of dissolved air in water. The dissolved air mass is supposed to be sufficiently low so that water properties are not influenced.

Total flow of latent and sensible heat in an unsaturated porous medium is given based on Philip and De Vries's theory (Philip and De Vries, 1957):

$$
Q=-\lambda_{\text {equ }} \nabla(T)+\left\lfloor\rho_{W} \mathbf{V}_{\text {vap }}+\rho_{v} \mathbf{V}_{\mathbf{a}}\right\rfloor h_{f g}+\left\lfloor C_{P W} \rho_{W} \mathbf{U}+C_{P V} \rho_{W} \mathbf{V}_{\text {vap }}+C_{P a} \rho_{a} \mathbf{V}_{\mathrm{a}}\right\rfloor\left(T-T_{0}\right)
$$

where $C_{P W}, C_{P V}$ and $C_{P a}$ are, respectively, the water, vapour and gas specific heat capacities per unit mass, $h_{f g}$ is the latent heat of vaporization of soil water and $\lambda_{\text {equ }}$ represents the Fourier heat diffusion coefficient of unsaturated mixture.

For the energy conservation of an unsaturated porous medium, we can write: 
$\frac{\partial \Phi}{\partial t}+\operatorname{div} Q=0$

$\Phi$ is the volumetric bulk heat content, which is defined by

$\Phi=C_{T}\left(T-T_{0}\right)+(n-\theta) \rho_{\text {vap }} h_{f g}$

In which the specific heat capacity $C_{T}$ is considered to be defined as:

$$
C_{T}=(1-n) C_{P S} \rho_{S}+\theta C_{P W} \rho_{W}+(n-\theta) C_{P V} \rho_{v a p}+(n-\theta) C_{P a} \rho_{a}
$$

The equilibrium equation can also be written as follows:

$$
\left(\sigma_{i j}-\delta_{i j} p_{g}\right)_{, j}+p_{g, j}+b_{i}=0
$$

Where $b_{i}$ is body force.

\section{Numerical modelling and Verification}

\subsection{Triaxial mechanical tests}

To verify the adequacy of the model, some triaxial testes have been modelled hereafter. The tests are conducted on a clay stone from the East region of France called 'Argilites de l'Est' (Homand et al. 2002; Chiarelli and Shao, 2002; Chiarelli 2000). The specimens were $40 \mathrm{~mm}$ in height and $20 \mathrm{~mm}$ in radius and the tests were carried out under isothermal and drained conditions. In order to validate model for different loading paths, triaxial compression, triaxial lateral extension as well as triaxial compression tests with unloading - reloading cycles are simulated. The triaxial compression tests are carried out under confining pressures of $p_{c o n}=0,5,10,20 \mathrm{MPa}$. Having considered the geometry and the loading condition of the tests, an axisymmetric configuration is adopted for the model.

Preliminary parameters for elastoplastic and damage behaviour were mainly taken from argillite parameters introduced in the literature (e.g. Gatmiri and Arson 2008; Homand et al. 2002; Chiarelli and Shao, 2002). First, a triaxial lateral extension test was back-analyzed to adjust the preliminary parameters so as to obtain parameters that yielded the best fit to the experimental result. The Final model parameters obtained are summarized in Table 1.

Plastic compressibility modulus of argillite, $\gamma_{H}$, is not directly available in the literature. Hence the following equation is used to calculate it:

$$
\gamma_{H}=\frac{1+e_{0}}{\lambda-\kappa}=49.32
$$


For the parameters of degradation of elastic stiffness $\alpha_{1}=0$ and $\alpha_{2}=32\left(1-v_{0}\right) / 15\left(2-v_{0}\right)$ are adopted. These values are based on theoretical estimations presented by Budiansky and O'Connell (1976) which are confirmed by Chiarelli's experimental results (Shao et al. 2006).

Table1: Parameters of the Model

\begin{tabular}{|c|c|c|c|}
\hline$E_{0}(M P a)$ & $v$ & $\kappa$ & $\kappa_{s}$ \\
\hline 11800 & 0.17 & 0.005 & 0.008 \\
\hline$\lambda_{0}$ & $r$ & $\beta$ & $k$ \\
\hline 0.028 & 0.06 & 0.0076 & 0.05 \\
\hline$P_{c 0}(M P a)$ & $P_{r e f}(M P a)$ & $M$ & $\gamma_{H}$ \\
\hline 16 & 1 & 2 & 49.32 \\
\hline$C_{0}(P a)$ & $C_{l}(\mathrm{~Pa})$ & $g$ & $\zeta$ \\
\hline $2.3 \mathrm{E}-4$ & $5.2 \mathrm{E}-3$ & -1.414 & 0.05 \\
\hline
\end{tabular}

Using the determined parameters, triaxial compression tests are simulated and the results of the present poro-plastic damage model and the experimental results together with the results of the elastodamage model (Arson, 2009) are depicted in Fig. 5 to Fig. 8. The results of the elastodamage model (from Arson's PhD dissertation) are included here to compare the capabilities of the plasto-damage and the elastodamage models implemented in $\Theta$-STOCK. It is clearly shown in Fig. 5 to Fig. 8 that much better stress-strain results are obtained with the current plastodamage model.

The value of the damage versus deviatoric stress for the plastodamage model is plotted in Fig. 9. For all confining pressures the lateral components of the damage are equal in both directions $\left(\Omega_{r r}=\Omega_{\theta \theta}\right)$, while axial component of damage is zero $\left(\Omega_{z z}=0\right)$. Therefore the radial damage component, $\Omega_{r r}$, is illustrated in Fig. 9. The corresponding results obtained with elastodamage model (Arson, 2009) are shown in Fig. 10. The mechanically induced damages by the plastodamage model are about 3 to 4 times greater than those of the elastodamage model. In 
addition, the propagation threshold of the damage is somewhat lower with the platodamage model.

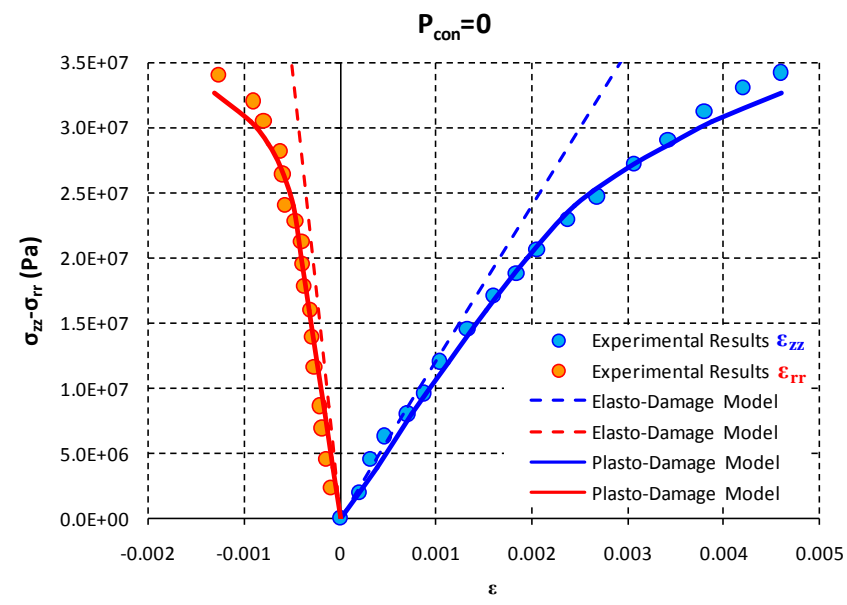

Figure 5: Stress-strain curve at zero confining stress $\left(p_{c o n}=0\right)$

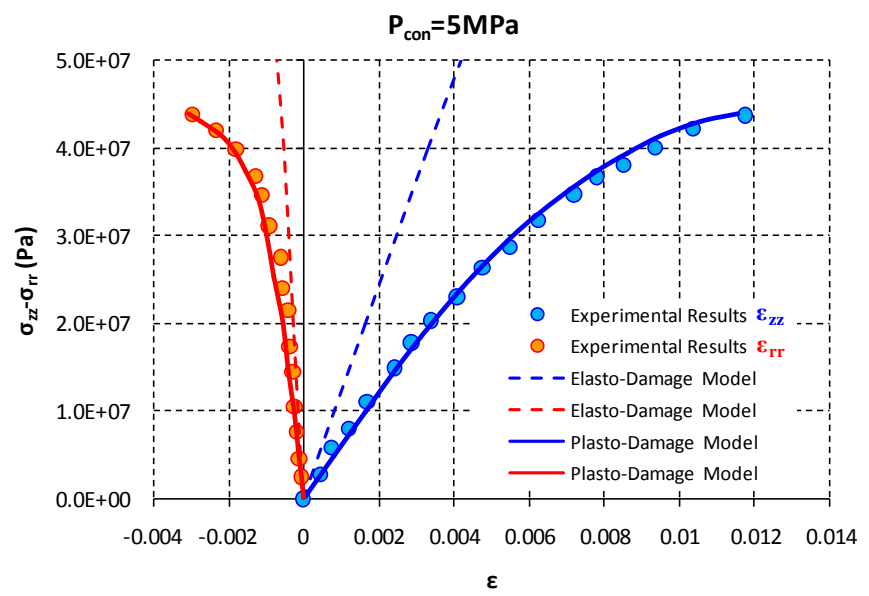

Figure 6: Stress-strain curve for the confining stress of $p_{\text {con }}=5 \mathrm{MPa}$ 


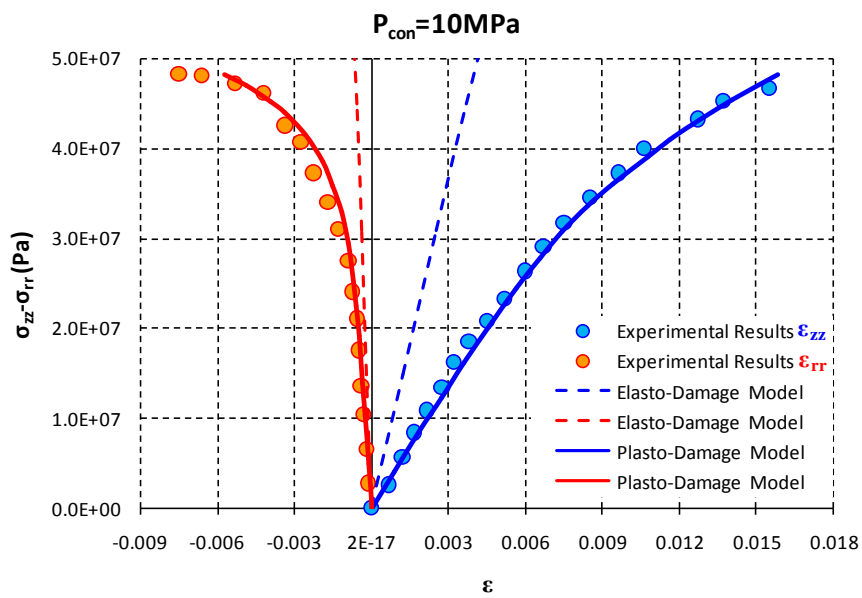

Figure 7: Stress-strain curve for the confining stress of $p_{c o n}=10 \mathrm{MPa}$

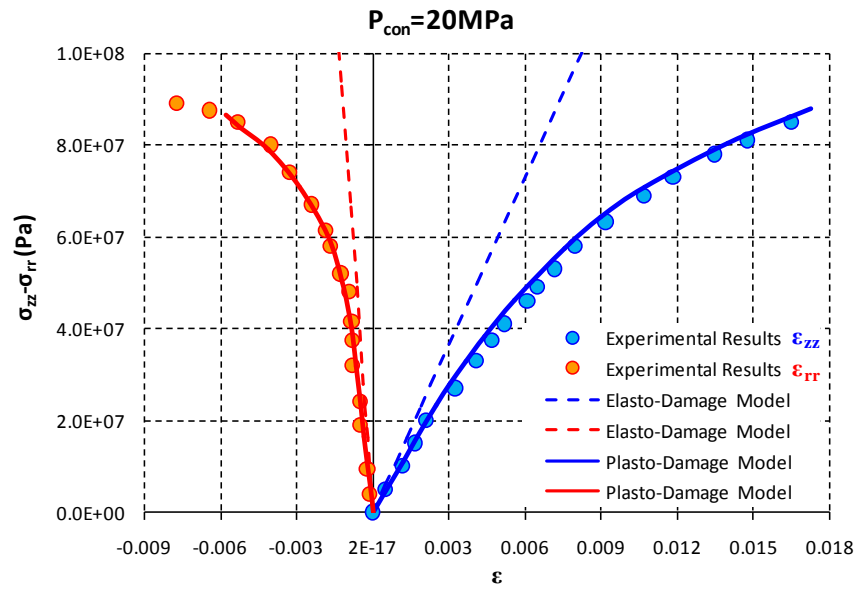

Figure 8: Stress-strain curve for the confining stress of $p_{c o n}=20 \mathrm{MPa}$ 


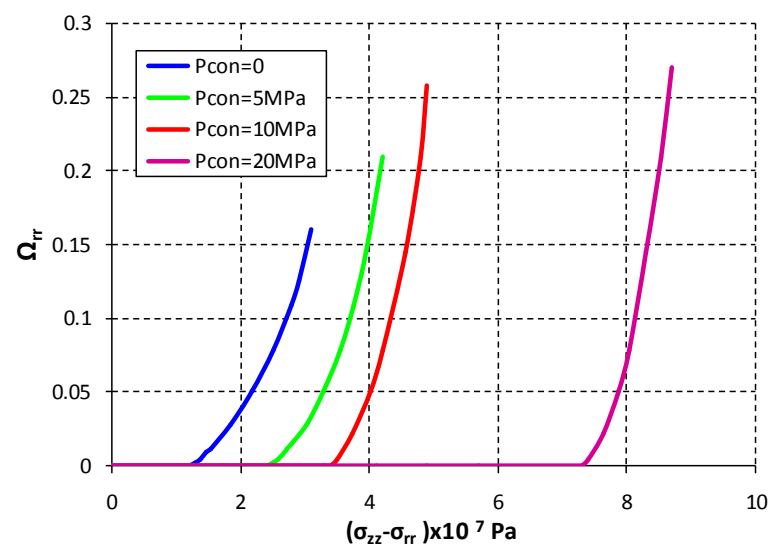

Figure 9: Evolution of radial damage with plastodamage model

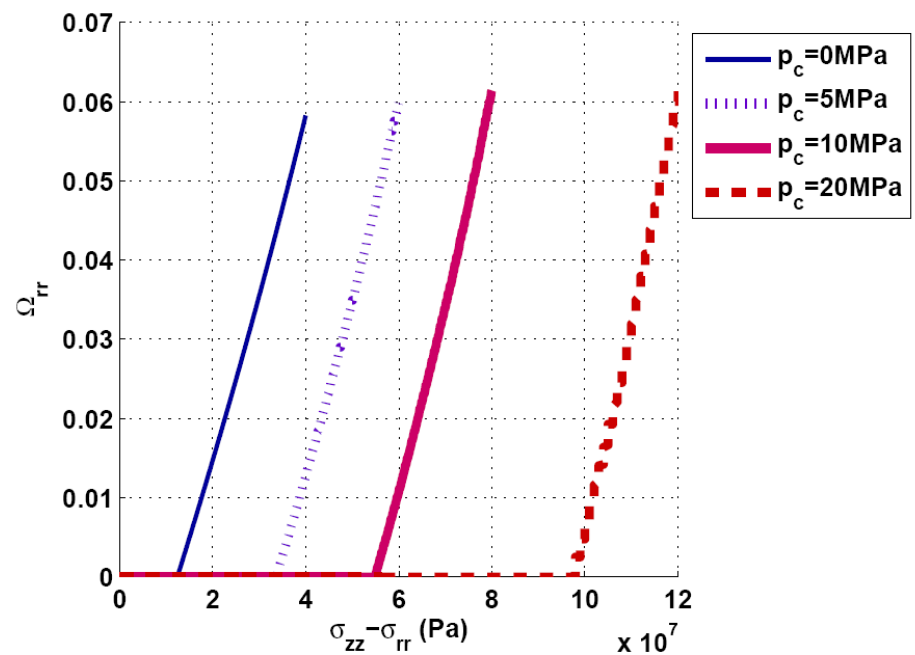

Figure 10: Evolution of radial damage with elastodamage model (Arson, 2009).

\subsubsection{Discussion}

In the presentation of the analysis results, the soil mechanics sign convention (compression positive) is adopted. As normally expected in a triaxial compression test, negative or tensile strains ( $\boldsymbol{\varepsilon}^{+}$in solid mechanics convention) have occurred in the lateral directions while axial strains are purely positive. In the formulation of the model, it is assumed that the damage growth is associated with the development of the generalized tensile strain. The zero axial damage $\left(\Omega_{z z}=0\right)$ is thus a reasonable result for all the confining pressures. Moreover, in a triaxial stress state with an axisymmetric configuration, equal tensile strains are 
calculated in the lateral directions, as a result, the lateral components of damage are equal $\left(\Omega_{r r}=\Omega_{\theta \theta}\right)$.

The strains of all directions have been significantly underestimated with the elastodamage model, which clearly indicates that the elastodamage model lacks a dissipative mechanism other than the tensile damage propagation. Such mechanism should generate inelastic deformations in all directions. In addition, an underestimated magnitude of strains with the elastodamage model has led to an underestimate of the damage intensity for all the confining pressures. In fact, the present plastodamage model has tackled the issue by introduction of the plastic flow which reproduces inelastic deformations as it is required.

Having used the same parameters for both models, the difference of the tensile strains is the only influential variable in the damage initiation threshold. The plastodamage model, on the other hand, calculates higher values of strains; consequently, a smaller value of deviatoric stress is expected to initiate fracturing of the medium with the plastodamage model. Hence the lower damage propagation threshold obtained by the plastodamage model is a consistent and admissible result.

One can see that the simulated stress-strain responses from the plastodamage model give a better match to the test data in all the confining stresses. These results can confirm the validity of the proposed model for simulation of behaviour of semi-brittle geomaterials.

It is, however, worth pointing out here that the previous applications of the elastodamage model have demonstrated its capability and reliability in the modelling of the brittle geomaterials like granite showing a negligible plastic behaviour (Arson and Gatmiri 2010, 2011, 2012). As a matter of fact, in such geomaterials, the brittle behaviour is a dominant response so that the elastodamage model can properly deal with the modelling of them.

\subsubsection{Modelling of degradation of the material stiffness}

Since no reliable damage measurement method is available, the degradation of elastic rigidity may be the only way to validate the damage part of model. In fact an appropriate model for argillites should be capable of capturing progressive degradation of the material stiffness. To evaluate the ability of the model to simulate this feature, a triaxial compression test with unloading-reloading cycles from Chiarelli's thesis is simulated hereafter. This simulation can also validate the proposed model in more complex loading conditions. Comparison of numerical results and experimental data are illustrated in Fig. 11. As can be seen, the model provided a reasonable result for the test with unloading cycles and it could reproduce degradation of elastic properties properly. 


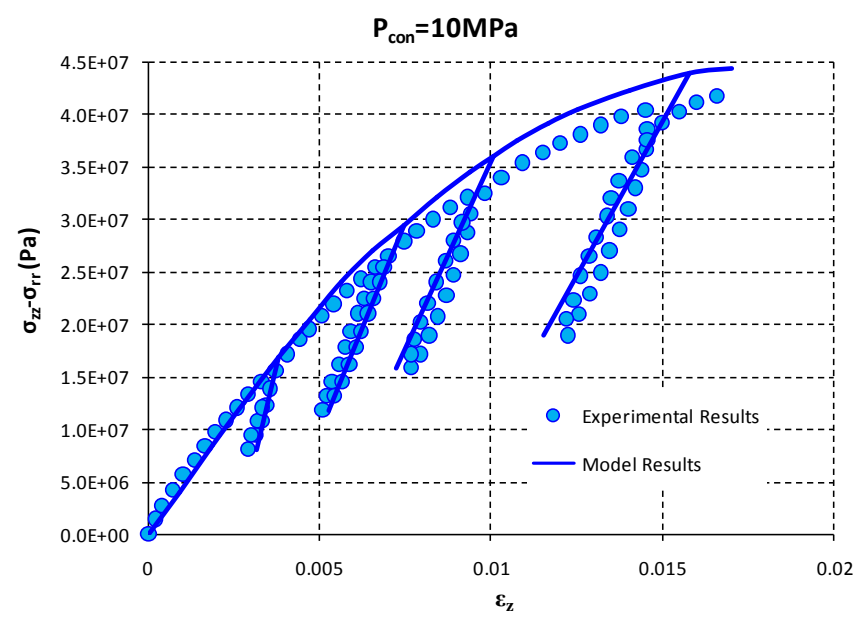

Figure 11: Comparison of model results and experimental data for unloading - reloading cycles

\subsection{Bentonite heating test}

Capillary-related characteristics of Barcelona Model and its variants are extensively discussed and verified in the literature; therefore it is decided to use a heating test for verification of coupled thermo-hydraulic features of the proposed model. In the framework of feasibility studies of geological disposal of high-level nuclear wastes, bentonites are being under intensive investigation (Pintado et al., 2002; Cótica et al. 2011; Keller et al. 2015; Pelayo et al. 2016) as potential materials for engineered barriers (EB). Evaluation and simulation of coupled thermo-hydro-mechanical response of bentonite buffers surrounding heatemitting waste canisters is one of active topics in the context. Therefore, hereafter we will simulate a heating experiment (Pintado et al., 2002) with the proposed plasto-damage model.

The test is performed by Pintado and co-workers in which two cylindrical specimens of $38 \mathrm{~mm}$ diameter and $76 \mathrm{~mm}$ height are prepared and a cylindrical heater of $50 \mathrm{~mm}$ height is placed between the two specimens. As explained in the reference paper, the unsaturated FEBEX bentonite samples are located in vertical position being surrounded with a latex membrane and a layer of heat insulating material. A controlled heat flow is applied by the heater to the hot end of specimens while a constant temperature of $30{ }^{\circ} \mathrm{C}$ is maintained at the other end of the samples (the cold end).

The material tested is a montmorillonite clay from Almería the South-East of Spain (FEBEX bentonite). Its geomechanical and geochemical properties are described extensively by Villar et al. 2012; Villar (1994) and ENRESA (1998). 
Montmorillonite composed more than 90 percent of FEBEX bentonite and variable amounts of quartz, plagioclase, K-feldspar, calcite and opal-CT (cristobalite-trydimite) are other constituents of this bentonite. $\mathrm{Ca}, \mathrm{Mg}, \mathrm{Na}$ and $\mathrm{K}$ respectively are Major exchangeable cations of FEBEX bentonite and the cation exchange capacity (CEC) varies from 96 to $102 \mathrm{meq} / 100 \mathrm{~g}$.

The size of $67 \pm 3$ percent of particles is smaller than $2 \mu \mathrm{m}$. The liquid limit of FEBEX bentonite is $102 \pm 4 \%$, the plastic limit is $53 \pm 3 \%$ and the specific gravity is $2.70 \pm 0.04$.

\subsubsection{Simulation of the test}

As the specimens are cylindrical, and the heat flux is symmetric around the central axis, it should be appropriate to adopt an axisymmetric formulation with a two-dimensional analysis. Due to the similarity of conditions for both the samples, only the lower sample is modelled here. A Finite Element mesh of dimensions of $r=19 \mathrm{~mm}$ and $z=76 \mathrm{~mm}$ is adopted for simulation which is depicted in Fig. 12a. The medium is discretized into 50 elements by a rectangular mesh with smaller elements for the zone nearer the heat source, where gradient of field variables is expected to be more significant. The dimensions of elements are adjusted to provide nodal data at distances of 20,38 and $60 \mathrm{~mm}$ from the upper boundary (heat source) because the experimental results of the same locations are reported in Pintado et al. (2002).
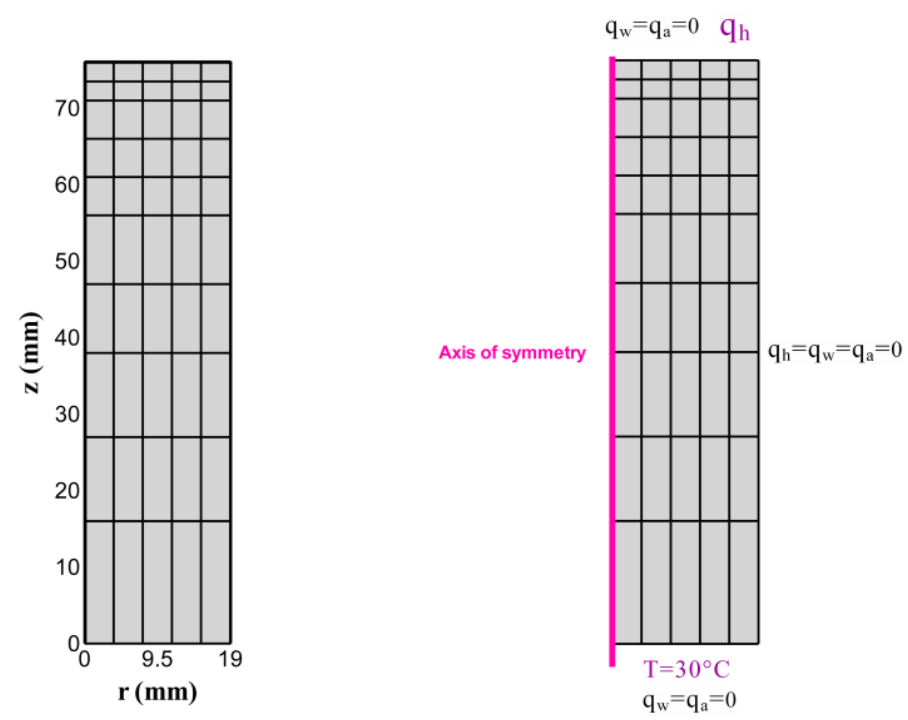

Figure 12: mesh and boundary conditions adopted for model 
The sample is heated through a natural or 'Neumann' boundary condition being applied to the nodes of the upper external boundary of the sample. The boundary conditions of the analysis are shown in Fig. 12b. A constant temperature of $\mathrm{T}=30^{\circ} \mathrm{C}$ is imposed on the lower nodes as 'Dirichlet' boundary condition while an adiabatic boundary condition is assumed for lateral sides of the model.

\subsubsection{Model parameters}

The elastoplastic and damage-related material properties used are summarized in Table 2. Damage parameters are adopted based on the recommendations of Arson 2009 which is originally inspired by the experimental results reported by Homand et al. (2002) and Chiarelli and Shao (2002).

Elastoplastic parameters are mainly chosen based on the works of research group of University of Catalunya on FEBEX project (Villar et al., 2008; Gens et al., 2009). The parameter of temperature-dependency of the plastic yield surface, $\gamma_{T}$, is taken from François and Laloui (2009). Plastic compressibility modulus $\gamma_{H}$ has been calculated in terms of coefficients of compressibility and void ratio through the following equation:

$$
\gamma_{H}=\frac{1+e_{0}}{\lambda-\kappa}=12.02
$$

Table 2: Elastoplastic and damage Parameters used in simulation

\begin{tabular}{|c|c|c|c|c|c|}
\hline \multicolumn{6}{|c|}{ Elastoplastic Properties } \\
\hline $\begin{array}{l}\alpha_{0}^{*}\left({ }^{\circ} \mathrm{C}^{-1}\right) \\
-1.5 \mathrm{E}-4 \\
\end{array}$ & $\begin{array}{c}\alpha_{1} \\
0 \\
\end{array}$ & $\begin{array}{c}\alpha_{2} \\
0 \\
\end{array}$ & $\begin{array}{c}\alpha_{3} \\
0 \\
\end{array}$ & $\begin{array}{c}\kappa_{i} \\
0.04 \\
\end{array}$ & $\begin{array}{c}\kappa_{s} \\
0.25 \\
\end{array}$ \\
\hline \multicolumn{6}{|c|}{ Elastoplastic Properties } \\
\hline $\begin{array}{c}p_{\text {geo }}(\mathrm{Pa}) \\
1.0 \mathrm{E} 5\end{array}$ & $\begin{array}{c}\lambda_{0} \\
0.94\end{array}$ & $\begin{array}{c}r \\
0.75\end{array}$ & $\begin{array}{c}\beta \\
0.05\end{array}$ & $\begin{array}{c}k \\
0.1\end{array}$ & $\begin{array}{c}\alpha\left({ }^{\circ} \mathrm{C}^{-1}\right) \\
0.0099\end{array}$ \\
\hline \multicolumn{6}{|c|}{ Elastoplastic Properties } \\
\hline $\begin{array}{c}P_{c 0}(M P a) \\
20 \\
\end{array}$ & $\begin{array}{c}P_{r e f}(M P a) \\
0.1\end{array}$ & $\begin{array}{l}M \\
1.5 \\
\end{array}$ & $\begin{array}{c}\gamma_{H} \\
12.02 \\
\end{array}$ & $\begin{array}{l}\gamma_{T} \\
2.1 \\
\end{array}$ & \\
\hline \multicolumn{6}{|c|}{ Damage Properties } \\
\hline $\begin{array}{l}C_{0}(\mathrm{~Pa}) \\
2.3 \mathrm{E}-4 \\
\end{array}$ & $\begin{array}{l}C_{l}(\mathrm{~Pa}) \\
5.2 \mathrm{E}-3 \\
\end{array}$ & $\begin{array}{c}g \\
-1.414 \\
\end{array}$ & $\begin{array}{l}\zeta \\
1 \\
\end{array}$ & & \\
\hline \multicolumn{6}{|c|}{ Initial Condition } \\
\hline $\begin{array}{c}\mathrm{S}_{\mathrm{w} 0} \\
0.63\end{array}$ & $\begin{array}{c}e_{0} \\
0.322 \\
\end{array}$ & $\begin{array}{c}T_{0}\left({ }^{\circ} \mathrm{C}\right) \\
22 \\
\end{array}$ & & & \\
\hline
\end{tabular}

The parameters for fluid flows and balance equations are listed in Table 3 . These parameters are chiefly also based on publications concerning the FEBEX project (Villar et al., 2008; Gens et al., 2009). Nevertheless, in view of basic differences 
between the formulation of CODE_BRIGHT and $\Theta-S T O C K$, we have had to determine some parameters indirectly based on the data presented in the reference papers. For instance, the following water retention curve is employed for the present model.

$$
S_{w}=\left(S_{w, s}-S_{w, r}\right)\left(1+\left(\alpha_{V G} S\right)^{n_{V G}}\right)^{-1+\frac{1}{n_{V G}}}+S_{w, r}
$$

The retention parameters $\alpha_{V G}=1.21 * 10^{-8} \mathrm{~Pa}^{-1}$ and $n_{V G}=1.98$ have been adopted, in a manner, to provide the best match with the water retention curve used by Gens et al. (2009).

In addition, in order to determine Fourier heat diffusion coefficient for unsaturated mixture, experimental data reported in Villar et al. (2008) as well as corresponding values in the present model for $\lambda_{\text {equ }}$ have been plotted in Fig. 13. The latter has been obtained using the equation 36 with parameters, $\lambda_{s}=0.94, \lambda_{w}=0.6, \lambda_{\text {vap }}=0.0258$, for thermal conductivities of the solid, water and gas phases, respectively.

$$
\lambda_{\text {equ }}=(1-n) \lambda_{s}+n S_{w} \lambda_{w}+\left(n-\theta_{w}\right) \lambda_{\text {vap }}+\left(n-\theta_{w}\right) \lambda_{a}
$$

Table 3: Input parameters for fluid flows and balance equations

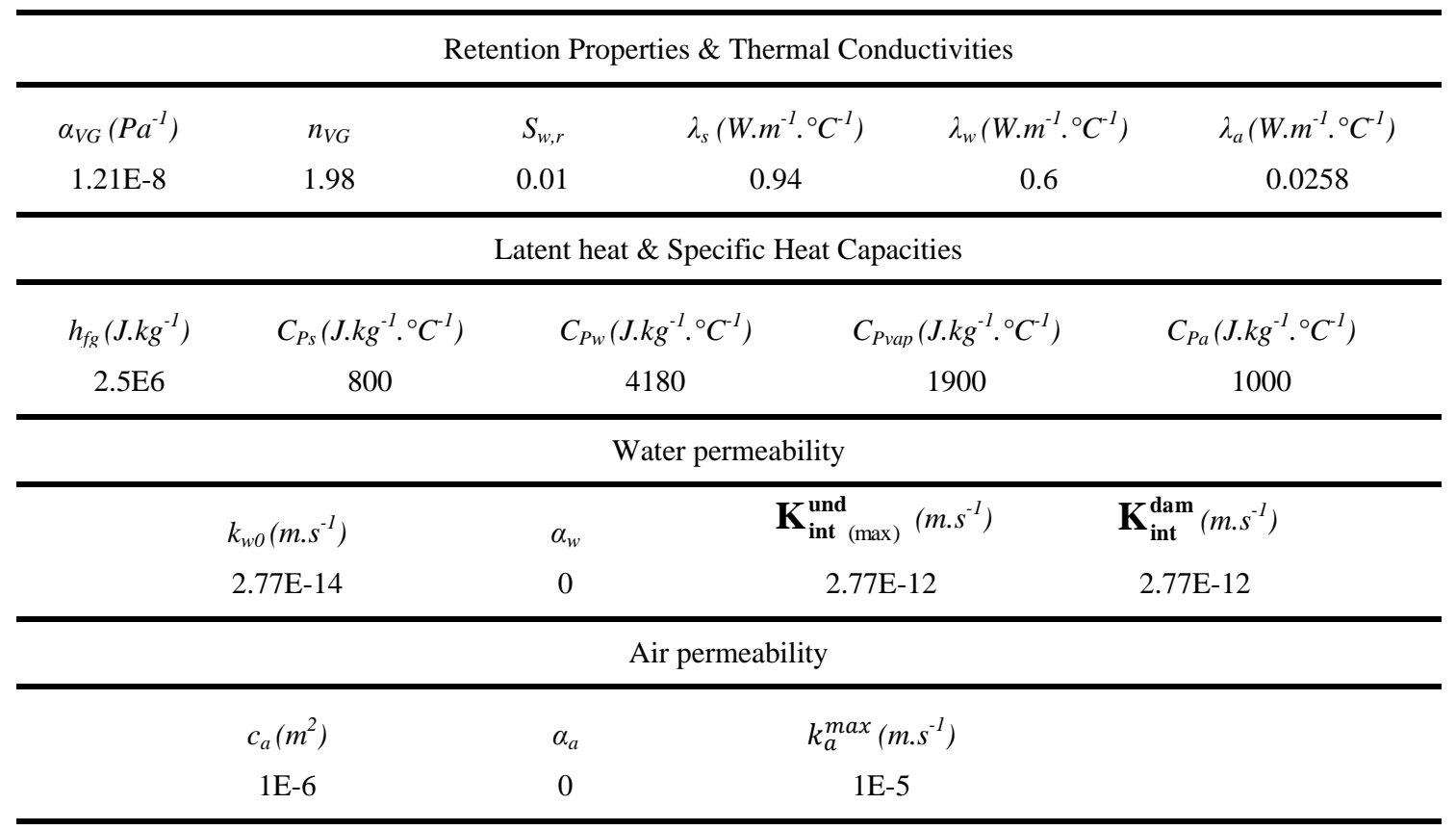




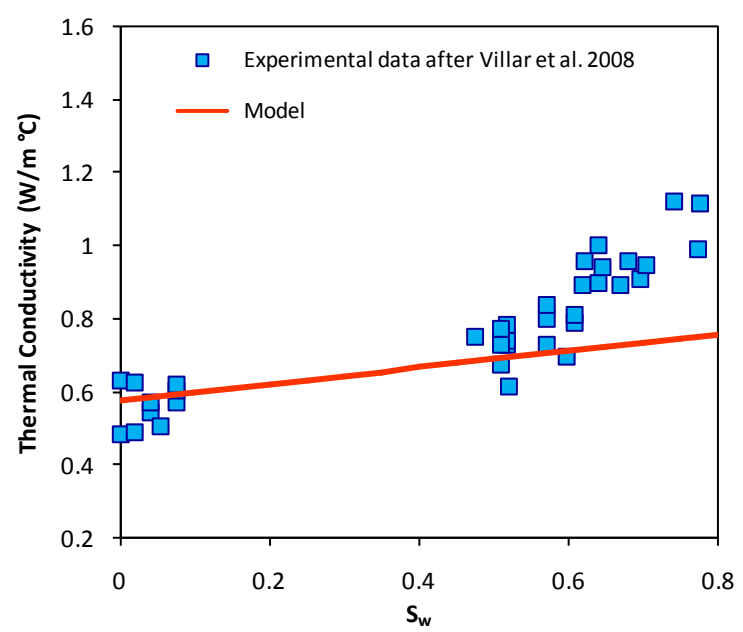

Figure 13: Thermal conductivity used in model compared with experimental data provided by Villar et al. 2008

\subsubsection{Results and discussion:}

Fig. 14 shows the evolution of temperature computed using the plasto-damage model which is compared with the measured ones by Pintado and co-workers. The results are illustrated at distances $\mathrm{x}=0,20,38,60,76 \mathrm{~mm}$ from the heat source. A quasi-steady regime of temperature (i.e. thermal balance) is practically attained 10 hours after the start of heating as it was observed in the experiment. In Fig. 15 isochrones of temperature obtained by analysis is compared with those from the test results and a good agreement is found.
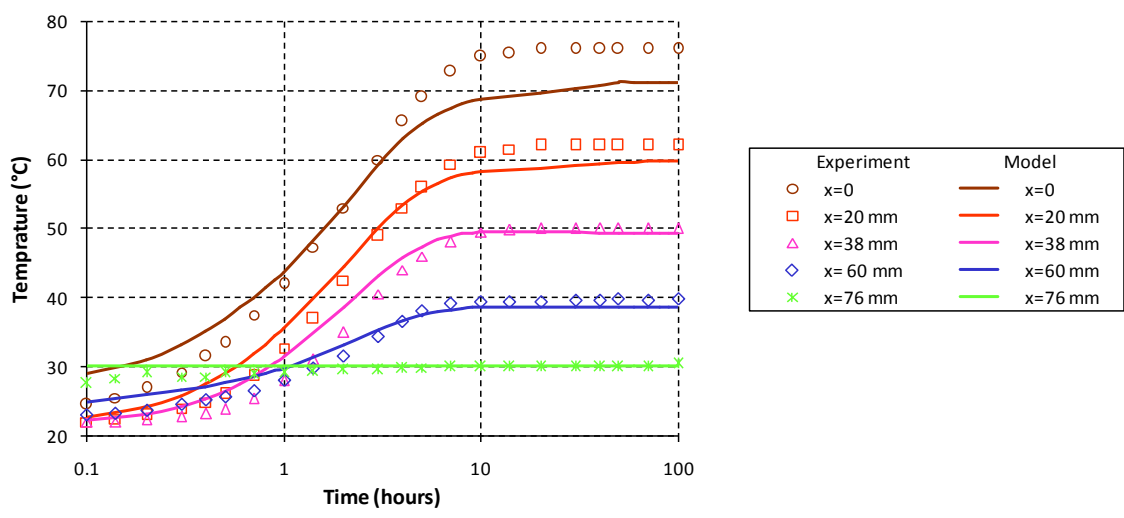

Figure 14: Evolution of temperature within the sample 


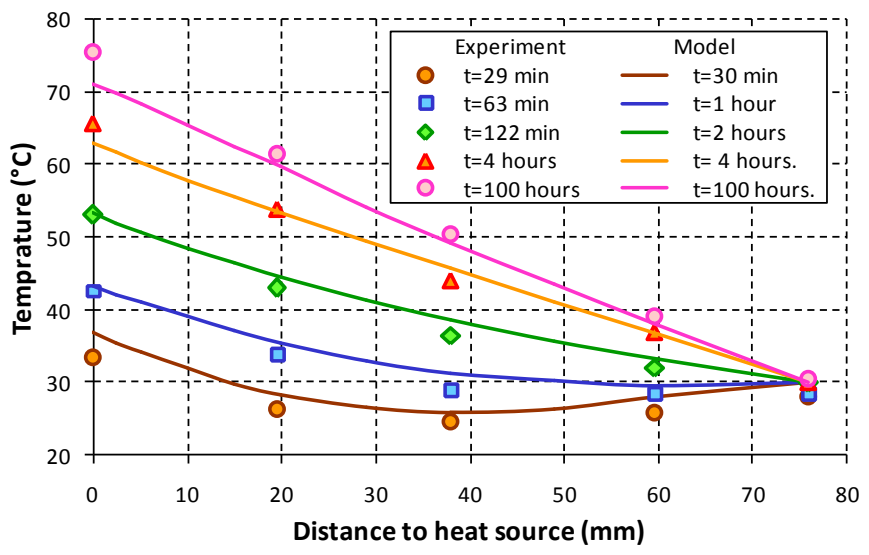

Figure 15: Isochrones of temperature

Water content values measured at the end of heating in two samples and corresponding result calculated by the model are depicted in Fig. 16. A reasonable overall match between the results is observed with a slight deviation at the cool end of the sample. It is worth noting that a water loss through the membrane and the membrane-heater contact area is reported in the reference paper which would be responsible for the mentioned difference.

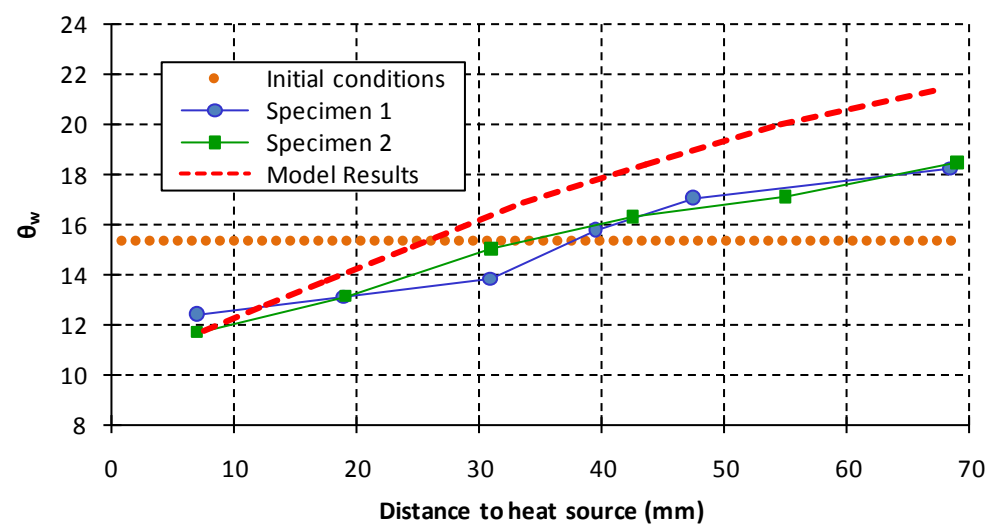

Figure 16: Water content values at the end of heating

Figs. 17a,b,c respectively show distributions of temperature, water content and suction within the sample using the proposed model. The results are presented for times, $\mathrm{t}=0.5,1,2,4,100$ hours after the commencement of the heating. As can be 
seen from these figures, during the early stages of the solution, the changes in temperature, water content and suction have initially occurred in the vicinity of upper boundary. As the analysis proceeds, the affected zone is expanded to the bottom boundary of the domain with further temperature rise. Nevertheless, the rate of variation of temperature is noticeably higher than those of the water content and the suction.

a)

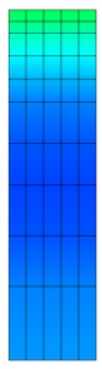

$t=30$ min

b)

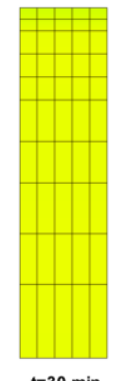

c)
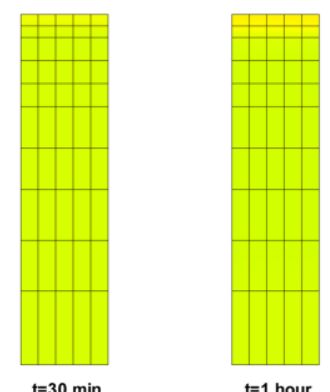

$\mathrm{t}=1$ hour

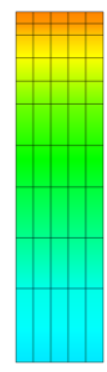

$t=2$ hours

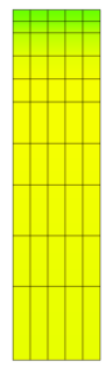

$t=2$ hours

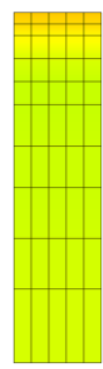

$t=2$ hours

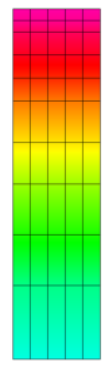

$t=4$ hours

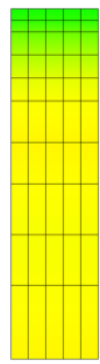

$t=4$ hours

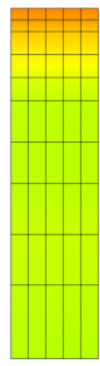

$\mathrm{t}=4$ hours

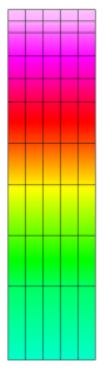

$t=100$ hours
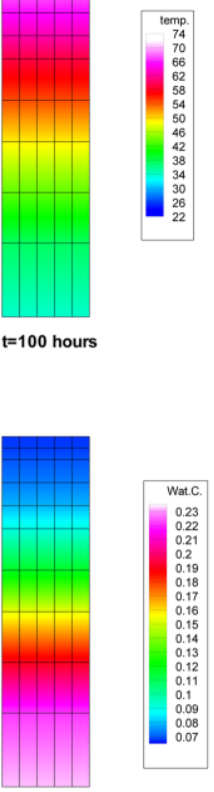

$t=100$ hours

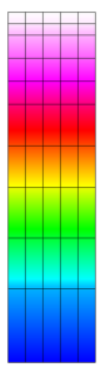

$t=100$ hours

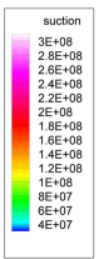

Figure 17: Distribution of a) temperature b) water content c) suction 
When the heat flux is applied to the model, the temperature of medium start increasing which give rise to evaporation near the heater. The vapour migrates vertically downwards within the specimen. As water vapour arrives at cooler region, a condensation front develops there over time. Through this process, a fraction of water is driven away from the hot end causing a decrease of the water content in the hot end and an increase of that in the cooler end. Also as can be seen in Fig. 17c, the suction is elevated in drier zone and diminished in the wetter side, due to the moisture transfer occurred.

Since the degree of saturation and the suction were not measured during the test and only a destructive method is used to measure the final water content, it is not possible to further verify the results obtained. However, overall it can be said that the simulation with proposed model has provided results which are physically reasonable and in accordance with the behaviour of unsaturated porous media. These results can confirm the validity of the proposed model for simulation of complex, coupled problems of geomaterials.

\section{Conclusion}

In the earlier sections of this paper in the introductory text, attention is focused on adoption of stress variables for the constitutive modelling of unsaturated porous media. Eventually, independent stress state variables including net stress and suction is adopted for formulation of model thanks to supporting experiments and physical appropriateness. Afterwards, framework of a new elastoplastic damage model is presented in which Barcelona Basic Model is extended to non isothermal state and is coupled to a damage formulation. It is assumed that damage growth is associated with the development of tensile strains and damage evolution law is derived by a normal dissipation scheme. Following the notion of preceding models of Gatmiri, a fully coupled formulation of fluid transfer laws and balance equation is employed and water intrinsic permeability tensor is split to undamaged and damaged parts.

The proposed model is implemented. To investigate capability of the model in mechanical domain, it is verified against the experimental results (triaxial tests) from the literature and the results of an elastodamage model. Afterwards the results of the model are verified against results of a heating test on FEBEX bentonite to assess the ability of model to predict the behaviour of an unsaturated massif hosting a heating source. Simulations with the proposed model have provided results which are in conformity with the reference temperature and saturation degree evolutions. The results given by model are also physically reasonable and in accordance with the behaviour of unsaturated porous media. These results confirm the validity of the proposed model for simulation of complex, coupled problems of geomaterials. 
Acknowledgements The authors gratefully acknowledge the financial support provided by Iran National Science Foundation (INSF) through grant No. 92015293, for the research presented in this paper. The first author is grateful to Dr Chloé Arson of Georgia Institute of Technology, for her assistance in various stages of this study and also to Dr Xavier Pintado for his helpful discussions and advice on experimental data. Their assistance is greatly appreciated.

\section{References:}

Abu Al-Rub, R. K.; Voyiadjis, G. Z. (2003): On the coupling of anisotropic damage and plasticity models for ductile materials. International Journal of Solids and Structures, vol. 40, pp. 2611-2643.

Ahangar-Asr, A., Javadi, A. A. and Khalili, N. (2015): An evolutionary approach to modelling the thermo-mechanical behaviour of unsaturated soils. International Journal for Numerical and Analytical Methods in Geomechanics, vol. 39, pp. 539-557.

Alizadeh, A.; Gatmiri, B. (2015): A New Elastoplastic Damage Model for Constitutive Modeling of Argillaceous Formations. $10^{\text {th }}$ International Congress on Civil Engineering, Tabriz, Iran.

Alizadeh, A.; Gatmiri, B. (2015): A Fully Coupled Thermo Hydraulic PoroPlasto-Damage Model for Semi-Brittle Geomaterials. $10^{\text {th }}$ International Congress on Civil Engineering, Tabriz, Iran.

Alonso, E. E.; Gens, A.; Josa, A. (1990): A constitutive model for partially saturated soils. Géotechnique, vol. 40, no. 3, pp. 405-430.

Arson, C.; Gatmiri, B. (2011): Numerical study of damage in unsaturated geological and engineered barriers. Physics and Chemistry of the Earth, vol. 36, pp. 1981-1989.

Arson C, Gatmiri B. (2012): Thermo-hydro-mechanical modeling of damage in unsaturated porous media: Theoretical framework and numerical study of the EDZ. International Journal for Numerical and Analytical Methods in Geomechanics, vol. 36, no.3, pp. 272-306.

Bishop, A. W. (1959): The principle of effective stress. Teknisk Ukeblad 39, Oct., 859-863.

Bishop, A. W.; Blight, G. E. (1963): Some aspects of effective stress in saturated and partially saturated soils. Géotechnique, vol. 13, no. 3, pp. 177-197. Blight, G.E. (1967): Effective stress evaluation for unsaturated soils. J. Soil Mech. Found. Div. Am. Soc. Civ. Engrs, vol. 93(SM2), pp. 125-148.

Chiarelli, A. S. (2000): Experimental investigation and constitutive modelling of coupled elastoplastic damage in hard argillites, Doctoral thesis (in French), University of Lille.

Chiarelli, A. S.; Shao, J. F. (2002): Modélisation élastoplastique couplée à l'endommagement anisotrope induit pour des argilites. Revue Francaise de Génie Civil, 6(1):115-130. 
Chiarelli, A. S.; Shao, J. F.; Hoteit, N. (2003): Modelling of elastoplastic damage behaviour of a claystone. International Journal of Plasticity, vol. 19, pp. 23-45.

Coleman, J. D. (1962): Stress-strain relations for partly saturated soils. Correspondence to Géotechnique 12(4):348-350.

Cordebois J. P.; Sidoroff, F. (1982) : Endomagement anisotrope en élasticité et plasticité. Journal de Mécanique théorique et appliquée, vol. 45-60 (special issue).

ENRESA, (1998): FEBEX. Bentonite: origin, properties and fabrication of blocks. Pulicación Te'cnica ENRESA, 05/98, Madrid.

François, B. ; Laloui, L. (2009) : ACMEG-TS: a constitutive model for unsaturated soils under non-isothermal conditions. International Journal for Numerical and Analytical Methods in Geomechanics, 32(16):1955-1988.

Hansen, N.R.; Schreyer, H.L. (1994): A thermodynamically consistent framework for theories of elastoplasticity coupled with damage. International Journal of Solids and Structures 31(3):359-389.

Fredlund, D. G.; Morgenstern, N. R. (1977): Stress state variables for unsaturated soils. J. Geotech. Eng. Div., ASCE, vol. 103(GT5), pp. 447-466. Fredlund, D. G. (2006): Unsaturated Soil Mechanics in Engineering Practice. Journal of Geotechnical and Geoenvironmental Engineering 132(3):286-321. Gallipoli, D.; Gens, A.; Sharma, R.; Vaunat, J. (2003): An elasto-plastic model for unsaturated soil incorporating the effects of suction and degree of saturation on mechanical behavior. Géotechnique, vol. 53, no. 1, pp. 123-135. Gatmiri, B.; Delage, P. (1997): A formulation of fully coupled thermalhydraulicmechanical behavior of saturated porous media - numerical approach. International Journal for Numerical and Analytical Methods in Geomechanics, vol. 21, no. 3, pp. 199-225.

Gatmiri, B.; Seyedi, M.; Delage, P.; Fry, F. F. (1997): A new suction-based mathematical model for thermo-hydro-mechanical behavior of unsaturated porous media. Proceedings of the Sixth International Symposium on Numerical Models in Geomechanics, NUMOG VI, Quebec, Canada, pp. 291-296.

Gatmiri, B.; Delage, P.; Cerrolaza, M. (1998): UDAM: powerful finite element software for the analysis of unsaturated porous media. Int J Adv Eng Software, vol. 29 , no. 1 , pp. 29-43.

Gatmiri, B. (2002): Framework of a nonlinear fully coupled thermo-hydromechanical behavior of unsaturated porous media. In Keynote lecture of the 3rd Iranian int. conference on geotechnical engineering and soil mechanics, Tehran, Iran, 9-11 December.

Gatmiri, B.; Hoor, A., (2007): Excavation effect on the thermo-hydromechanical behaviour of a geological barrier. Physics and Chemistry of the Earth, vol. 32 (8-14), pp. 947-956. 
Gatmiri, B.; Arson, C. (2008): $\Theta$-stock, a powerful tool for thermohydromechanical behaviour and damage modeling of unsaturated porous media. Computers and Geotechnics, vol. 35, no. 6, pp. 890-915.

Gatmiri, B.; Hemmati, S.; Arson, C.; Amirzehni, E. (2010a): A multiphase analysis for environmental impact assessment with $\theta$-stock finite element program. Journal of Multiscale Modeling, vol. 2, pp. 23-68.

Gatmiri, B.; Maghoul, P.; Duhamel, D. (2010b): Two-dimensional transient thermo-hydro-mechanical fundamental solutions of multiphase porous media in frequency and time domains. International Journal of Solids and Structures 47:595-610.

Gens, A.; Sánchez, M.; Guimarães, L. do N.; Alonso, E.E. ; Lloret, A.; Olivella, S.; Villar, M.V.; Huertas, F. (2009): A full-scale in situ heating test for high-level nuclear waste disposal: observations, analysis and interpretation. Géotechnique, vol. 59, pp. 1-23.

Houlsby, G.T.; Amorosi, A.; Rojas, E. (2005): Elastic moduli of soils dependent on pressure: a hyperelastic formulation. Géotechnique 55(5):383-392. Homand, F.; Chiarelli, A. S.; Hoxha, D. D. (2002): Caractéristiques physiques et mécaniques du granite de la Vienne et de l'argilite de l'Est. Revue Française de Génie Civil 6(1):11-20.

Hutter, K.; Laloui, L.; Vuillet, L. (1999): Thermodynamically based mixture theory models for saturated and unsaturated soils. Mechanics of Cohes. Frict. Mat, vol. 4, pp. 295-338.

Jabbari, E.; Gatmiri, B. (2007): Thermo-Poro-Elastostatic Green's Functions for Unsaturated Soils. CMES: Computer Modeling in Engineering \& Sciences, vol. 18, pp. 31-44.

Jommi, C. (2000): Remarks on constitutive modelling of unsaturated soils. In Experimental evidence and theoretical approaches in unsaturated soils (eds A. Tarantino and C. Mancuso.), pp. 139-153. Rotterdam: Balkema.

Ju, J. W. (1989): On energy-based coupled elastoplastic damage theories: constitutive modelling and computational aspects. International Journal of Solids and Structures, vol. 25, no. 7, pp. 803-833.

Kachanov, L.M. (1958): On the Creep Fracture Time, Izv. Akad. Nauk USSR Otd. Tech, vol. 8, pp. 26-31 (in Russian)

Kachanov, M. (1992): Effective elastic properties of cracked solids: critical review of some basic concepts, Applied Mechanics Reviews, vol. 45, no. 8, pp. 304-335.

Khalili, N.; Khabbaz, M. H. (1998): A unique relationship for the determination of the shear strength of unsaturated soils. Géotechnique, vol. 48, no. 5, pp. 681687.

Laloui, L.; Nuth, M. (2009): On the use of the generalised effective stress in the constitutive modelling of unsaturated soils. Computers and Geotechnics, vol. 36, pp. 20-23. 
Matyas, E. L.; Radhakrishna, H. S. (1968): Volume change characteristics of partially saturated soils. Géotechnique 18(4):432-448.

Nuth, M,; Laloui, L. (2008): Effective stress concept in unsaturated soils: clarification and validation of a unified framework. International Journal for Numerical and Analytical Methods in Geomechanics, vol. 32, no. 7, pp. 771-801. Ortiz, M. (1985): A constitutive theory for the inelastic behaviour of concrete. Mechanics of Materials, vol. 4, pp. 67-93.

Philip, J.R.; De Vries, D. A. (1957): Moisture movement in porous materials under temperature gradients. Transactions in American Geophysics, vol. 38, pp. 222-232.

Pintado, X.; Ledesma A.; Lloret, A. (2002): Backanalysis of thermohydraulic bentonite properties from laboratory tests. Engineering Geology, vol. 64, pp. 91115.

Shao, J.F.; Jia, Y.; Kondo, D.; Chiarelli, A.S. (2006): A coupled elastoplastic damage model for semi-brittle materials and extension to unsaturated conditions. Mechanics of Materials, vol. 38, pp. 218-232.

Sheng, D.; Sloan, S. W.; Gens, A.; Smith, D. W. (2003): Finite element formulation and algorithms for unsaturated soils. Part I: Theory. International Journal for Numerical and Analytical Methods in Geomechanics, vol. 27, pp. 745-65.

Sheng, D.; Smith, D. W.; Sloan, S. W.; Gens, A. (2003): Finite element formulation and algorithms for unsaturated soils. Part II: Verification and application. International Journal for Numerical and Analytical Methods in Geomechanics 27:767-90.

Sheng, D.; Gens, A.; Fredlund, D. G.; Sloan, S. W. (2008): Unsaturated soils: From constitutive modelling to numerical algorithms. Computers and Geotechnics, vol. 35, pp. 810-824.

Tarantino, A.; Mongiovi, L. (2000): Experimental investigations on the stress variables governing unsaturated soil behavior at medium to high degrees of saturation. Experimental Evidence and Theoretical Approaches in Unsaturated Soils; Proceedings of an International Workshop, Trento, pp. 3-18.

Tarantino, A.; Mongiovi, L.; Bosco, G. (2000): An experimental investigation on the independent isotropic stress variables for unsaturated soils. Géotechnique, vol. 50, no. 3, pp. 275-282.

Villar, M.V., (1994): Modelling and validation of the thermal-hydraulicmechanical and geochemical behaviour of the clay barrier. Final Report 19911994. CIEMAT, Madrid.

Villar, M.V.; Sánchez, M.; Gens, A. (2008): Behaviour of a bentonite barrier in the laboratory: Experimental results up to 8 years and numerical simulation. Physics and Chemistry of the Earth, vol. 33, pp. S476-S485. 
Villar , M.V.; Gómez-Espina, R.; Gutiérrez-Nebot, L. (2012): Basal spacings of smectite in compacted bentonite. Applied Clay Science vol. 65-66, pp. 95105.

Voyiadjis, G.Z.; Deliktas, B. (2000): A coupled anisotropic damage model for the inelastic response of composite materials. Computer Method in Applied Mechanics and Engineerings, vol. 183, pp. 159-199.

Wheeler, S. J., Sharma, R. J. \& Buisson, M. S. R. (2003): Coupling of hydraulic hysteresis and stress-strain behaviour in unsaturated soils.

Géotechnique, vol. 53, no.1, pp. 41-54.

Xiong, Y., Zhang, S., Ye, G., Zhang, F. (2014): Modification of thermo-elastoviscoplastic model for soft rock and its application to THM analysis of heating tests, Soils and Foundations, vol. 54, no. 2, pp. 176-196.

Shuku, T.; Murakami, A.; Nishimura, S.; Fujisawa, K.; Nakamura K., (2012): Parameter identification for Cam-clay model in partial loading model tests using the particle filter, Soils and Foundations, vol. 52, no. 2, pp. 279-298.

B. Dehandschutter, S. Vandycke, M. Sintubin, N. Vandenberghe, P.

Gaviglio, J.-P. Sizun, L. Wouters (2004): Microfabric of fractured Boom Clay at depth: a case study of brittle-ductile transitional clay behaviour, Applied Clay Science, vol. 26, no. 1-4, pp. 389-401.

F. Zhang, S.Y. Xie, D.W. Hu, J.F. Shao, B. Gatmiri (2012): Effect of water content and structural anisotropy on mechanical property of claystone, Applied Clay Science, vol. 69, pp. 79-86.

Qiong Wang, Yu-Jun Cui, Anh Minh Tang, Pierre Delage, Behrouz Gatmiri, Wei-Min Ye (2014): Long-term effect of water chemistry on the swelling pressure of a bentonite-based material, Applied Clay Science, vol. 87, pp. 157162.

A. Gaudin, S. Gaboreau, E. Tinseau, D. Bartier, S. Petit, O. Grauby, F. Foct, D. Beaufort (2009): Mineralogical reactions in the Tournemire argillite after insitu interaction with steels, Applied Clay Science, vol. 43, no. 2, pp. 196-207.

H.G Montes, J Duplay, L Martinez, S Escoffier, D Rousset (2004): Structural modifications of Callovo-Oxfordian argillite under hydration/dehydration conditions, Applied Clay Science, vol. 25, no. 3-4, pp. 187-194.

V. Magnenet, C. Auvray, G. Francius, A. Giraud (2011): Determination of the matrix indentation modulus of Meuse/Haute-Marne argillite, Applied Clay Science, vol. 52, no. 3, pp. 266-269.

M Elie, P Faure, R Michels, P Landais, L Griffault, L Mansuy, L Martinez (2004): Effects of water-cement solutions on the composition of organic compounds leached from oxidized Callovo-Oxfordian argillaceous sediment, Applied Clay Science, vol. 26, no. 1-4, pp. 309-323.

María D. Alba, Pablo Chain, M. Mar Orta (2009): Chemical reactivity of argillaceous material in engineered barrier: Rare earth disilicate formation under subcritical conditions, Applied Clay Science, vol. 43, no. 3-4, pp. 369-375. 
Liange Zheng, Jonny Rutqvist, Hui-Hai Liu, Jens T. Birkholzer, Eric

Sonnenthal (2014): Model evaluation of geochemically induced

swelling/shrinkage in argillaceous formations for nuclear waste disposal, Applied Clay Science, vol. 97-98, pp. 24-32.

Xiaojun Zhu, Jingong Cai, Guoqi Song, Junfeng Ji (2015): Factors

influencing the specific surface areas of argillaceous source rocks, Applied Clay Science, vol. 109-110, pp. 83-94.

M. Pelayo, E. García-Romero, M.A. Labajo, L. Pérez del Villar (2016):

Evidence of montmorillonite/Fe-rich smectite transformation in the Morrón de Mateo bentonite deposit (Spain): Implications for the clayey barrier behaviour, Applied Clay Science, In Press, Corrected Proof.

L.F. Cótica, V.F. Freitas, I.A. Santos, M. Barabach, F.J. Anaissi, R.Y.

Miyahara, P.W.C. Sarvezuk (2011): Cobalt-modified Brazilian bentonites:

Preparation, characterisation, and thermal stability, Applied Clay Science, vol.

51, no. 1-2, pp. 187-191.

Lukas M. Keller, Lorenz Holzer, Philippe Gasser, Rolf Erni, Marta D.

Rossell (2015): Intergranular pore space evolution in MX80 bentonite during a long-term experiment, Applied Clay Science, vol. 104, pp. 150-159. 